\title{
Coordination et connaissance : éléments sur les apports autrichiens à l'analyse de la firme
}

\section{Michel Renault}

\section{(2) OpenEdition \\ 12 Journals}

Édition électronique

URL : http://journals.openedition.org/ei/844

DOI : $10.4000 /$ ei.844

ISSN : 2553-1891

Éditeur

Association Économie et Institutions

Édition imprimée

Date de publication : 1 décembre 2004

Pagination : 5-52

ISSN : 1775-2329

\section{Référence électronique}

Michel Renault, «Coordination et connaissance : éléments sur les apports autrichiens à l'analyse de la firme », Économie et institutions [En ligne], 5 | 2004, mis en ligne le 31 janvier 2013, consulté le 24 avril 2019. URL : http://journals.openedition.org/ei/844 ; DOI : 10.4000/ei.844 


\title{
Coordination et connaissance : Eléments sur les apports autrichiens à l'analyse de la firme ${ }^{1}$
}

\author{
Michel RENAULT2
}

Le fil directeur de l'ouvrage de B.Coriat et O.Weinstein (1995), était, comme ils le soulignaient eux-mêmes, la distinction entre la firme comme organisation et la firme comme institution.

L'objet de notre article est centré sur ces deux dimensions fondamentales et interdépendantes de la firme à partir de la mise en évidence des apports de deux approches qui ne sont évoquées que de façon marginale dans l'ouvrage: la tradition autrichienne d'une part, et d'autre part la tradition institutionnaliste.

Il nous semble que des développements et des réinterprétations récents ont permis de renouveler les interrogations de ces deux courants de pensée et d'ouvrir des pistes de réflexion pour fonder des approches renouvelées de la firme. Pour le courant autrichien on citera par exemple et sans être exhaustif les travaux de N.J.Foss, P.Garrouste, B.J.Loasby, T.Fu-Lai Yu ou de R.Langlois et pour les institutionnalistes ceux de L.Bazzoli, V.Dutraive, de B.F.Kaufman, ainsi que les nôtres.

Notre perspective ne prétendra pas examiner tous les aspects de ces approches de la firme, encore parfois embryonnaires, mais sera centrée sur les problématiques essentielles et connexes de la coordination et de la connaissance (et donc sur les dimensions cognitives de la firme) qui constituent, nous semble t-il, le cœur de ces approches et leur spécificité. Le parallèle entre ces deux approches, qui diffèrent par bien des aspects, nous semble ainsi justifiée par la proximité de leurs problématiques et la possibilité d'interrogations croisées.

Une des faiblesses relevée des approches contractualistes est en effet de limiter la connaissance à de l'information et les problèmes d'interaction et de coordination à des questions d'asymétries d'information devant être résolues par des incitations adéquates.

1 Cet article a été présenté initialement au colloque "Economie de la firme: quelle nouveautés?" Annecy 17-18 Avril 2003. La présente version tient compte des remarques des deux rapporteurs de la revue que je tiens à remercier pour leurs remarques pertinentes et constructives. Je demeure bien entendu responsable des erreurs et omissions que pourrait comporter ce texte.

2 CREM-Université de Rennes I, michel.renault@univ-rennes1.fr

Economie et Institutions $-n^{\circ} 5-2^{e}$ semestre 2004 
D'un autre côté, les approches néo-institutionnalistes ${ }^{3}$, bien qu'empruntant le concept de transaction à la tradition institutionnaliste, n'envisagent la firme que comme un dispositif fonctionnel alternatif au marché et qui ne se définit que par rapport à lui. Dans ce cadre, le problème de la compatibilité des représentations et des cadres cognitifs d'interprétation des agents est largement négligé.

Les autrichiens soulignent ainsi que la connaissance n'est pas seulement dispersée, mais aussi subjectivement dispersée, en particulier du fait de cadres d'interprétation différents. De la même façon l' approche institutionnaliste met l'accent sur ce qu'il est convenu d'appeler aujourd'hui des "modèles mentaux partagés" ou des "dispositifs cognitifs collectifs" qui permettent d'assurer la convergence des anticipation des agents et la coordination des comportements, des actions et des plans. En ce sens, une firme ne peut se résumer à une sommation de contrats (y compris de contrats dits "relationnels" évoqués par R.Gibbons pour appréhender les phénomènes de culture et de confiance dans les organisations) mais constitue une structure de coordination des actions qui transite par des aspects proprement organisationnels (division du travail, localisation des responsabilités...) et des aspects institutionnels (représentations, routines, coutumes, langage...). Ces aspects peuvent, par exemple, s'incarner concrètement dans l'analyse de la relation de travail.

A cet égard nous partageons l'opinion de Bazzoli et Dutraive [1998b] qui soulignent que pour les institutionnalistes, et c'est également vrai pour les autrichiens, l'opposition entre institution et organisation n'est pas pertinente. Toute firme est en effet une institution au sens de Commons qui souligne que l'institution, à la fois, contraint et libère l'action individuelle. On peut ainsi définir la notion d'institution comme: "[...] l'organisation des attitudes que nous portons tous en nous, c'est à dire les attitudes organisées des autres qui contrôlent et déterminent la conduite" [Mead 1963]. Les autrichiens soulignent également le rôle de coordination des routines, des capacités et des construits cognitifs partagés. La

3 Nous réserverons le terme néo-institutionnalisme à la tradition issue des travaux de R.Coase et dont O.Williamson est la figure emblématique. L'approche que nous qualifions ici d'institutionnaliste s'inscrit dans la perspective du "vieil" institutionnalisme américain issu des travaux de T.Veblen et surtout de J.R.Commons. Nous considérerons également dans le cadre de cet "institutionnalisme" les travaux récents, en particulier dans le domaine de la sociologie, qui se réfèrent au pragmatisme, qui est selon nous intimement lié à l'institutionnalisme américain et ne peut en être dissocié, c'est le cas par exemple des travaux de H.Joas ou J.Beckert.

6 Economie et Institutions $-\mathrm{n}^{\circ} 5-2^{\mathrm{e}}$ semestre 2004 
firme peut ainsi être considérée comme un ordre spontané (institution organique) et comme un ordre délibéré (institution pragmatique). Les aspects cognitifs apparaissent donc bien fondamentaux pour ces approches. Nous voudrions, de plus, mettre en exergue, comme nous avons déjà pu le faire par ailleurs [Renault1999], les aspects communicationnels qui sous tendent ces processus cognitifs. Dans ce cadre nous serons amené a mettre l'accent, aussi bien en ce qui concerne l'approche autrichienne que pour l'approche institutionnaliste, sur les fondements de ses analyses. En particulier nous insisterons pour les autrichiens sur les apports de A.Schütz, récemment redécouverts et réinterprétés, et pour les institutionnalistes sur la philosophie pragmatique et ses incarnations dans les sciences sociales à travers les contributions de J.Dewey et G.H.Mead, également réévaluées récemment. Cela nous conduira a approfondir les relations entre les deux courants de pensée. Dans les deux cas, l'action humaine et son appréhension apparaissent comme des enjeux fondamentaux ${ }^{4}$. Nous présenterons donc d'abord les théories de l'action qui sous tendent les conceptions autrichiennes et institutionnalistes (I) et nous soulignerons ensuite les relations entre action et institutions (II), enfin nous présenterons les dimensions créatives de l'action en particulier à travers la référence à l'entrepreunariat (III).

\section{I-Action et connaissance}

\section{-le problème de la coordination}

Le point de départ d'une théorie autrichienne de la firme est sans conteste la question de la coordination des plans d'actions individuels [Garrouste 2002]. La question de la connaissance apparaît alors intimement liee à celle de la coordination à travers le point de vue subjectiviste. Cette question de la connaissance est elle même intimement liée à la conception de l'action humaine et des impératifs cognitifs qui sous tendent l'action. Les autrichiens considèrent en effet que la connaissance n'est pas seulement dispersée entre les individus, mais qu'elle est subjectivement dispersée car les individus ne partagent pas les mêmes cadres cognitifs, les mêmes théories, les mêmes routines...Ainsi: "un tel point de vue subjectiviste a plusieurs conséquences et justifie que le problème essentiel de l'analyse économique soit d'étudier la façon dont les individus parviennent à coordonner leurs plans d'action (...). En

4 De nombreuses confrontations ont déjà été effectuées entre ces deux formes d'"institutionnalisme" ( voir par exemple W.J.Samuels (ed.) [1989], en particulier Boettke). Notre approche se place sous l'angle particulier des dimensions cognitives et communicationnelles de l'action.

Economie et Institutions $-n^{\circ} 5-2^{e}$ semestre 2004 
effet, si les individus ne peuvent avoir une connaissance parfaite de ce qui détermine les actions des autres, la mise en compatibilité de ces actions devient alors un problème essentiel"[Dulbecco et Garrouste 2000 p.89]. Cette perspective est sans doute ce qui constitue une des caractéristique majeure du "courant" autrichien ${ }^{5}$, qui selon PJ.Boettke [2002] marque un tournant épistémique/cognitif dans 1' analyse économique, ce qui nous semble pertinent pour appréhender les firmes.

Cette façon d'envisager les choses est essentiellement différente de celle de la théorie dominante de la firme fondée sur la théorie de l'information qui considère principalement des asymétries d'information, et on pourrait dire essentiellement des asymétries de répartition (même si le problème des asymétries d'acquisition et de traitement n'est pas totalement négligé via la rationalité limitée qui est cependant plus souvent revendiquée que réellement utilisée) ${ }^{6}$. Le problème de la coordination des actions, des comportements et des plans est réduit à un problème d'alignement des intérêts divergents d'individus, via des contrats qui permettent de lutter contre les phénomènes usuels d'opportunisme, d'aléa moral, de sélection averse... qui sont au cœur de la théorie moderne des organisations, celle-ci étant essentiellement si l'on suit J.Crémer, une théorie de l'information.

Ainsi, une des faiblesses relevée des approches contractualistes est de limiter la connaissance à de l'information et les problèmes de coordination et d'interaction à des questions d'asymétries d'information devant être résolues par des incitations

5 Le courant autrichien est loin d'être homogène et, si l'on suit Boettke [2002], ne s'est réellement défini comme courant autonome que de façon relativement récente, en particulier avec la génération d'économistes formés dans les années 1950, principalement M.Rothbard, I.Kirzner ou L.Lachmann (se plaçant eux même dans la continuité des travaux de Menger, Mises ou Hayek). Les caractéristiques de ce courant listées par Boettke sont diversifiées selon les auteurs et peuvent être mis en évidence à travers la référence à des perspectives partagées: individualisme méthodologique, subjectivisme, nature entrepreunariale du processus de marché, temporalité de l'action et des processus productifs...Pour notre perspective la référence au subjectivisme apparaît essentielle. Outre les auteurs clairement reconnus comme "autrichiens", d'autres auteurs s'inscrivent, au moins partiellement, dans des perspectives ouvertes par ces auteurs, c'est le cas par exemple de N.J.Foss, U.Witt, T.Fu-Lai Yu, R.N.Langlois...Notre travail n'étant pas un pur travail d'historien de la pensée nous avons choisi de les regrouper sous cet intitulé générique de courant autrichien, même si nous sommes conscient de consentir à une approximation. Nous ne retenons cependant dans ces travaux que ce qui se réfère explicitement à un héritage autrichien.

6 voir à ce propos Foss [2003]

8 Economie et Institutions $-\mathrm{n}^{\circ} 5-2^{\mathrm{e}}$ semestre 2004 
adéquates. Comme le souligne N.J.Foss [1998a p.15]: "[...] the issue is not merely one of incentive compatibility which is the predominant form that plan consistency takes in the modern economics of organization; it is also one of discovering knowledge (e.g. overcoming sheer ignorance) and aligning mental frameworks" (nous soulignons).

En particulier, une des critiques autrichiennes porte sur la représentation des situations d'interaction en terme de théorie des jeux qui obscurcirait des problèmes fondamentaux dans une perspective subjectiviste: comment les joueurs sont-ils amenés à connaître les gains des autres ? Les stratégies disponibles ? Comment peuvent-ils avoir la même vision concernant ces gains ? La même vision sur chacun d'entre eux ? Comment savent-ils quel type de jeu, et quel jeu particulier, ils jouent ?...Comme le souligne N.J.Foss, la perspective de la théorie des jeux ne permet pas réellement d'aborder le problème de la connaissance puisque dans ce cadre celle-ci n'est pas considérée comme étant subjectivement dispersée. Il y a ainsi une confusion entre ce qui existe objectivement et ce qui est subjectivement perçu, ce qui avait été largement mis en avant par Hayek [voir Foss 1998a p.18]. Cette question peut en particulier mettre en jeu la conception de l'entrepreneur en tant que porteur d'une "vision", d'une représentation mentale du marché, qui doivent être communiquées et partagées.

\section{-La conception subjective de l'action humaine}

L'approche autrichienne part d'une autre conception fondée sur un certain nombre de propositions dont découlent des conséquences. Nous voudrions ici souligner certains points concernant notre perspective sans prétendre à l'exhaustivité:

-Tout d'abord, le concept central de l'approche autrichienne est celui de "processus de marché" (market process) qui implique une rivalité active et une perspective en terme de déséquilibre [Kirzner 1997]. La perspective néo-classique conceptualise au contraire la concurrence en terme de cohérence des décisions de maximisation des consommateurs et des producteurs (ce qui est logique compte tenu de l'absence de dispersion subjective de la connaissance). Hayek a ainsi largement mis en avant le fait que la concurrence parfaite implique l'absence de toute activité réellement concurrentielle. Dans la perspective autrichienne, la concurrence est avant tout un mécanisme pour découvrir, mobiliser et disséminer de la connaissance dispersée [Foss 1998b]. On sait que dans ce cadre le système des prix a un rôle essentiel à jouer. On reconnaît cependant 
aujourd'hui qu'au delà du système des prix, l'organisation a un rôle à jouer dans un tel processus.

-L'approche autrichienne repose sur une conception subjectiviste qui est au cœur du problème de la "connaissance dispersée" comme nous l'avons déjà souligné; cela mérite cependant d'être précisé tant ce point est essentiel. En effet comme le soulignait I.Kirzner [1973] nous ne savons pas ce que nous ne savons pas. De plus, l'un des inspirateurs de la conception autrichienne de l'action , A.Schütz ${ }^{7}$, soulignait que la connaissance dépend de la façon dont les individus "définissent la situation". Un point intéressant à noter est que Schütz s'inspirait de la fameuse citation du sociologue W.I.Thomas, proche du pragmatisme, selon lequel, si les individus définissent des situations comme réelles, elles sont réelles dans leurs conséquences [Fu-Lai Yu1999 p.29] ${ }^{8}$. I.Kirzner [1979 p.137] affirmait ainsi que "Things are what the acting people think they are". De même, les individus savent que des événements imprévus, des nouveautés, peuvent surgir mais ils ne peuvent pas prévoir le contenu de ces événements ou nouveautés. A cela il faut ajouter le fait que les individus sont en possession de cadres interprétatifs ou de "modèles mentaux"9 différents ce qui rend ces problèmes plus aigus. Dans un tel cadre, l'entrepreneur est amené à exercer une vigilance particulière (entrepreneurial alertness) pour découvrir, mobiliser et utiliser la connaissance afin de réaliser un profit [Dulbecco et Garrouste 2000 p.91-92] ${ }^{10}$. La représentation ou la conception mentale que l'entrepreneur peut se faire du marché ou de la situation apparaît ainsi comme une dimension importante.

Le subjectivisme autrichien est ainsi fondé comme le mettent en évidence Garrouste et Dulbecco [2000 p.88] sur quatre éléments:

1-l'analyse intègre la façon dont l'intellect (ou l'esprit) se construit. La capacité de traitement de l'information est

${ }^{7}$ Le philosophe et sociologue A.Schütz a été influencé par, et a influencé, les économistes autrichiens. Son rôle d'influence a récemment été réactualisé et de nombreux travaux d'auteurs autrichiens méritent d'être réexaminés à cette aune. Son œuvre nous semble essentielle puisqu'elle présente de nombreuses intersections avec la perspective pragmatique qui sous tend l'institutionnalisme. Sur les rapports de Schütz avec la courant autrichien voir: C.Prendergast [1986], Foss [1996a], A.Oakley [2000], J.E.Mote [2001], P.Kurrild-Klitgaard [2003]. La Review of Austrian Economics a consacré un numéro spécial à Schütz en 2001 [n²-3].

8 Nous aurons l'occasion de décliner ce point à plusieurs reprises dans des contextes différents

9 Pour reprendre les termes de Denzau et North [1994]

10 Le "leadership cognitif" évoqué par U.Witt [1998] peut aussi être évoqué à ce propos

10 Economie et Institutions $-\mathrm{n}^{\circ} 5-2^{\mathrm{e}}$ semestre 2004 
limitée, en particulier par des facteurs contextuels. Les individus dans un tel cadre sont amenés à suivre des règles de comportement.

2-la capacité cognitive des individus s'inscrit dans le "temps réel", qui se définit lui même comme la perception subjective du temps. L'apprentissage est ainsi un élément central de l'analyse $\mathrm{du}$ comportement individuel puisqu'il permet de faire face au flux temporel qui implique l'incertitude, l'émergence, l'irréversibilité...La rationalité est ainsi largement apprise (et donc contextuelle), ce qui est très important dans le cadre de notre perspective.

3-Les individus sont des individus actifs. Contrairement à certaines représentations, en particulier celle de la théorie de l'agence ou de la théorie des incitations, les individus ne s'adaptent pas passivement à l'environnement, mais au contraire peuvent réduire l'incertitude intrinsèque en construisant des institutions "(...) qui rendent plus prévisibles les contraintes que constituent le comportement des autres, et plus généralement leur environnement" [Dulbecco et Garrouste 2000 p.89]. Ici encore cet aspect nous semble devoir être mis en exergue car il rejoint la perspective institutionnaliste.

4-le quatrième élément est la subjectivité de la connaissance déjà évoqué: différents individus auront une connaissance différente. Ainsi, contrairement à l'homogénéité réactionnelle de la théorie des organisations dominante, différents agents agiront différemment dans un contexte identique du fait de différences dans la perception et le traitement de 1'"information". L.Lachmann a ainsi souvent insisté sur le fait que des individus différents dans un même contexte n'auront pas les même perceptions.

Dans ce cadre, la coordination des plans d'action dans un environnement factuel et comportemental incertain constitue un problème essentiel pour l'analyse économique. La firme, ou plus généralement l'organisation, apparaît ainsi comme une solution au problème de la connaissance dispersée pour paraphraser P.Garrouste [1995] ${ }^{11}$.

11 Dans le cadre de notre perspective, la présentation de la firme dans une perspective autrichienne faite par S.Longuet [2001] nous semble particulièrement pertinente.

Economie et Institutions $-n^{\circ} 5-2^{e}$ semestre 2004 


\section{-La construction sociale de la réalité}

Les éléments précédents renvoient à une théorie autrichienne de l'action, souvent implicite, que certains cherchent à expliciter [Foss 1996, Fu-Lai Yu 1999, 2001, 2003a, 2003b par exemple]. Cette théorie de l'action renvoie in fine aux travaux du philosophe et sociologue A.Schütz qui avait largement insisté sur l'importance des phénomènes de perception et d'interprétation des "données" du monde physique par les individus.

A.Schütz considérait que le marginalisme autrichien, issu des travaux de Menger poursuivi par Von Mises manquait d'une théorie de la compréhension intersubjective qui apparaît essentielle si on aborde les questions de la coordination et de l'ordre spontané, et plus généralement celui de la connaissance [Voir Foss 1996 p.75]. Partant de la notion d'action sociale issue des travaux de M.Weber ainsi que de sa conception de l'idéal type, Schütz a exploré ce que ses "héritiers" P.Berger et T.Luckmann [1966] ont appelé la "construction sociale de la réalité". En ce sens une action est sociale à partir du moment où elle prend en compte le comportement des autres et ou un sens subjectif lui est attribué. Schütz insistait ainsi sur ce qu'il appelait les structures intersubjectives de signification (intersubjective structures of meaning) qui permettent, si on reprend les termes de W.I.Thomas, d'aboutir à des définitions communes des situations, sans lesquelles il ne peut y avoir ni intercompréhension, ni coordination des actions. Dans son ouvrage de 1952 "The sensory order", Hayek se plaçait dans une perspective similaire, il écrivait en effet: "[...] it is [...] the views people have formed of each other and of the things, which form the true element of social structures" [1952 p.59 cité dans Foss 1996a]. Le problème de la coordination, lié aux dimensions subjectives de la connaissance, est donc avant tout un problème de coordination des "vues" ou des "définitions de la situation" que se donnent les individus. Dans "The sensory order", Hayek a développé une théorie de l'esprit permettant de comprendre comment les individus perçoivent et interprètent la réalité. Il a en particulier développé l'idée que la perception est fondée sur des dispositions de l'esprit à la catégorisation et à la classification, l'esprit en tant que système de classification se formant progressivement par expérience et processus d'essai et d'erreur. En particulier ce qui est significatif pour notre propos est que les perceptions et les interprétations en découlant sont sujettes à une révision constante en fonction de la disjonction entre les catégories existantes et des événements nouveaux ${ }^{12}$. D'une façon générale, l'ouvrage met en avant l'aspect relationnel de la perception [Voir

12 Voir Loasby [2001]

12 Economie et Institutions $-\mathrm{n}^{\circ} 5-2^{\mathrm{e}}$ semestre 2004 
Kaisla 2002], et l'esprit apparaît comme un produit "culturel" [Hörwitz 2000 p.24-25]. Avant Denzau et North, et la psychologie cognitive actuelle, Hayek mettait en avant les notions de "carte" (map) ou de "modèle" pour décrire l'ordre mental. Cette perspective est complémentaire de celle de Schütz qui met en avant les aspects sociaux et relationnels de la formation de ces "modèles mentaux"13.

A.Schütz a souligné, dans la perspective de Weber, que toute action présente deux faces [Bange 1992 p.77]:

-une face externe: une action est un ensemble observable de gestes, de postures, de modifications physiques...

-une face interne: elle met en jeu des processus cognitifs: perceptions, définition des buts, décision, valeurs, savoir social...

C'est cet aspect interne qui compose la signification de l'action. L'action est donc liée à une interprétation qui permet à l'acteur de conférer un sens à son action, de la même façon un coacteur interprétera, donnera un sens à l'action de l'autre ${ }^{14}$. Comme le dit A.Schütz dans le cadre d'une interaction un "(...) partenaire n'a connaissance ni du projet qui précède l'action de l'acteur, ni du contexte unitaire dans lequel elle s'inscrit. Il ne connaît que cette portion de l'action de l'acteur qui s'est manifestée à lui, c'est à dire l'exécution de l'acte qu'il a observé ou les phases passées de l'action qui est encore en cours.(...) Pour comprendre ce que moi, l'acteur, je voulais dire par mon action, il devrait partir de l'acte observé et construire à partir de là le motif-en-vue-de sous jacent pour lequel j'ai fait ce qu'il a observé" [Schütz 1962 p.24-1987 p.31; cité par Bange 1992 p.78]. On comprend bien à travers cela que l'enjeu de la coordination des actions est bien la compréhension, la reconstruction, du sens des actions. La coordination des actions impose en effet une interprétation commune de la réalité rendant possible une compréhension intersubjective.

13 B.J.Loasby a souligné que la subjectivité chez Hayek n'exclut pas l'intersubjectivité qui est un thème central chez Schütz. En particulier Hayek soutenait qu'une similarité d'expériences promeut une similarité de configuration ou de perception au niveau de l'individu ou de l'espèce [Loasby 2004]

14 Ainsi plus récemment pour D.Llerena [1997 p.361]: "Au delà des routines organisationnelles et des procédures mémorisées, les comportements et les actions des individus sont avant tout guidés par leurs interprétations. C'est l'interprétation de la réalité qui "crée" les problèmes et appelle à entreprendre des actions" (nous soulignons). 
Selon P.Bange, la coordination comporte ainsi deux aspects [1992 p.104]:

-la dépendance réciproque des décisions

-la convergence d'intérêts que les actions réciproques orientées ont pour but de réaliser

Le premier problème à résoudre est celui d'une attribution de sens identique conduisant à une définition partagée de la situation et donc à une inter-compréhension. Dans ce cadre, les typifications, qui représentent une connaissance (ou un savoir) sociale commune, apparaissent comme essentielles dans la coordination. Les typifications permettent à deux partenaires d'avoir des attentes réciproques qui seront mutuellement compatibles, des "attentes de comportement typique". Pour Schütz les agents diposent en effet, à tout moment donné du temps, d'un stock de connaissance disponible (knowledge at hand) ${ }^{15}$ qui leur sert de schéma d'interprétation de leurs expériences passées et présentes et déterminent leurs anticipations des choses à venir [Fu-Lai Yu 2001 p.53]. Une telle perspective est évidemment très proche de l'approche de G.H.Mead parlant d'attente de comportement généralisé, ce qu'on retrouve également chez l'économiste J.R.Commons, nous y reviendrons. On peut remarquer que la notion de typification est également proche de la notion de convention chez D.K.Lewis en tant que celle-ci génère un système d'attentes réciproques.

Dans cette perspective A.Schütz, comme G.H.Mead, met en avant un processus de réciprocité des perspectives qui rend intelligible la compréhension mutuelle et la coordination des actions. La réciprocité des perspectives repose sur la possibilité que chacun des individus a de se mettre à la place de l'autre et de voir la situation par ses yeux. Comme l'affirme P.Bange: "La "thèse générale des perspectives réciproques" est une construction théorique qui rend possible l'inter-subjectivité, sur laquelle repose la compréhension, la circulation des savoirs et l'interaction. Elle permet de comprendre que la connaissance que je prends du monde est "dès l'origine, intersubjective et socialisée" (Schütz 1987 p.16)" [1992 p.115]. La coordination, cependant, ne nécessite pas que la réciprocité des perspectives, il faut également qu'il y ait une réciprocité des

15 A.Schütz écrivait ainsi qu'on peut concevoir qu'il y a de multiples réalités selon les cadres interprétatifs mis en œuvre en fonction des buts poursuivis. Selon lui: "Toute interprétation est fondée sur une réserve d'expériences antérieures, qui sont nos propres expériences et celles transmises par nos parents et professeurs. Elles fonctionnent comme un cadre de référence sous la forme d'une connaissance disponible" [1987 p.105 cité par Bange 1992 p.21]. Cette perspective est très proche du pragmatisme.

14 Economie et Institutions $-n^{\circ} 5-2^{e}$ semestre 2004 
motivations. Pour Schütz: "Si j'imagine, en projetant mon acte, que tu le comprendras (c'est à dire que tu reconnaîtras mon intention) et que cette compréhension t'incitera à réagir, de ton côté, d'une certaine manière, j'anticipe que les motivations en-vue-de ma propre activité deviendront les motivations à-cause-de ta réaction et vice versa" [Schütz 1964 p.14 cité par Bange 1992 p.115]. Le principe de réciprocité des motivations de Schütz constitue, selon Bange [Ibid.] un autre nom pour désigner mon acceptation de subordonner mon action à la réalisation de ton but parce que la réalisation de ton but me rapproche de la réalisation du mien. Ces éléments constituent nous semble t-il les fondements d'une appréhension renouvelée de l'action humaine au cœur des apports autrichiens et que mettent en évidence les travaux récents tentant de constituer une "théorie autrichienne de la firme". Dans une large mesure, ces fondements trouvent des correspondances dans l'approche pragmatiqueinstitutionnaliste de l'action.

\section{-Les fondements comportementaux de l'approche institutionnaliste}

De la même façon que l'action chez les autrichiens est appréhendée par l'intermédiaire des références aux aspects cognitifs et sociaux, issus en particulier des travaux de Schütz, l'action chez les institutionnalistes, et plus spécifiquement chez J.R.Commons, doit être envisagée en faisant référence aux approches qui la sous tendent . Ainsi, les travaux des philosophes pragmatiques, notamment ceux de J.Dewey et G.H.Mead, apparaissent incontournables ${ }^{16}$. Ils établissent de plus un lien avec A.Schütz conduisant à un certain nombre de rapprochements entre perspective autrichienne et institutionnaliste sur les plans de la cognition et de l'action.

Un point de départ pertinent pour appréhender la prise en compte de l'action chez Commons est de se placer dans le cadre d'une perspective "relationnelle" ou "transactionnelle". Dans leur ouvrage de 1949 [1973] "The knowing and the known", J.Dewey et A.Bentley ont établi une distinction entre trois type d'approche:

-l'approche "auto-actionnelle" (self-actional)

-l'approche "interactionnelle" (interactional

-l'approche "transactionnelle" (transactional)

16 Sur les relations entre pragmatisme et institutionnalisme voir par exemple Renault [1992] et plus récemment Kilpinen [2003]

Economie et Institutions $-\mathrm{n}^{\circ} 5-2^{\mathrm{e}}$ semestre 2004 
Sans entrer dans les détails [voir Emirbayer 1997, Khalil 2003, Renault 2004], on peut dire pour simplifier que la conception auto-actionnelle correspond aux approches normatives (ou déterministes: le comportement est déterminé par des contraintes sociales), la conception interactionnelle correspond aux approches rationnelles (le comportement correspond à un calcul d'optimisation). Ces deux conceptions ont selon Dewey et Bentley la caractéristique d'opérer sur des "données", par exemple pour la conception rationnelle, familière en économie, les choix et les contraintes sont donnés et l'action répond à un "programme". Dans ce cadre, et contrairement à la perspective autrichienne développée précédemment, le contexte d'action, les significations et les interprétations des agents, c'est à dire les dimensions subjectives de l'action, sont négligés.

L'approche transactionnelle développée par Dewey et Bentley apparaît au contraire comme "relationnelle" et rejette le fait qu'on puisse poser des entités discrètes pré-données comme des points de départ de l'analyse. Les individus sont ainsi inséparables du contexte transactionnel; leur action dépend en effet des "définitions de la situation" qu'ils donnent [Emirbayer 1997, Dewey et Bentley 1973]. Comme l'ont souligné Dewey et Bentley, l'action de "nommer" n'est pas neutre et interagit avec la réalité, le "nommant" et le "nommé" sont en interaction [Dewey et Bentley 1973, p.131]. La définition donnée de la notion de transaction est la suivante: "where systems of description and naming are employed to deal with aspects and phases of action, without final attribution to "elements" or other presumptively detachable or independent "entities," "essences," or "realities," and without isolation of presumptively detachable "relations" from such detachable "elements." [Dewey et Bentley 1973, p.133]. Une telle perspective met bien en avant l'importance de la définition (sociale) de la réalité évoquée par l'approche de Schütz.

L'approche de J.R.Commons a été largement créditée pour avoir développé le concept de transaction; cependant cette notion s'inscrit chez lui dans une perspective plus large qui correspond à celle évoquée par Dewey et Bentley. Ces derniers le créditent en effet dans "The knowing and the known", même si c'est de façon limitative et partielle ${ }^{17}$. Une transaction chez Commons renvoie en effet à un

17 Ils écrivent ainsi en note: "Dewey's early employment of the word "transaction" was to stress system more emphatically than could be done by "interaction." (See his paper "Conduct and Experience" in Psychologies of 1930. [Worcester, Mass.] Compare also his use of "integration" in Logic, the Theory of Inquiry.) The beginnings of this attitude may be found in his paper "The Reflex Arc Concept in Psychology" (1896). Bentley's treatment of political events was of the transactional type in his The Process of Government

16 Economie et Institutions $-n^{\circ} 5-2^{e}$ semestre 2004 
contexte d'action qui fait que les individus pris en compte sont inséparables du contexte transactionnel. Cela renvoie également à ce que Commons appelait une psychologie négociationnelle (negotational) dont il attribuait la paternité à J.Dewey, il faut cependant également souligner la correspondance étroite entre cette approche et celle développé par G.H.Mead [Albert et Ramstad 1999, Renault 1997, 1999]. On peut essayer de résumer et de reconstruire la conception psychologique de Commons et du pragmatisme, sans être exhaustif, en retenant les éléments pertinents pour notre approche.

Tout d'abord chez Commons, les acteurs sont des acteurs sociaux doté d'un "esprit institutionnalisé" qui oriente leurs perceptions, leurs processus de délibération et leurs décisions. L'origine de cet "esprit institutionnalisé" ou de ces "cadres cognitifs partagés" est à rechercher dans la nature communicationnelle de la socialité évoquée par G.H.Mead, l'esprit institutionnalisé de Commons est en correspondance avec l'autrui généralisé de G.H.Mead [Renault 1999, Albert et Ramstad 1999]. La capacité d'identification, de prise de rôle, des individus et leurs interactions permanentes avec les autres les amène, via des processus d'habituation et d'imitation, à adopter des règles générales de conduite et des représentations communes de la réalité, ce que l'on peut qualifier d'"images". La proximité avec la perspective de Schütz et certains travaux de "style" autrichien [Fazio 2003] récents est significative [ Par exemple Witt 1999]. Dans ce cadre, comme le soulignait N.Wiley l'esprit "[...]is a kind of public square [...] the members of which are in constant conversation" [cité par Emirbayer 1997 p.297]. Cette perspective renvoie également à l'analyse développée par A.Smith dans la théorie de sentiments moraux qui apparaît comme un point de départ des travaux de Mead et des sociologies interactionnistes ultérieures comme celle de E.Goffman. Dans une telle perspective la connaissance porte sur des relations

(Chicago, 1908), though, of course, without the use of that name. John $\boldsymbol{R}$. Commons has used the word comparably in his Legal Foundations of Capitalism (New York, 1924) to describe that type of economic inquiry in which attention centers on the working rules of association rather than on material goods or human feelings. George H. Mead's "situational" is often set forth in transactional form,"(nous soulignons) [Dewey et Bentley 1973, p.133]. Commons [1934 p.73] écrivait ainsi: "These individual actions are really trans-actions, that is actions between individuals as well as individual behavior. It is this shift from commodities, individuals and exchange to transactions and working rules of collective action that marks the transition to the institutional school of economic thinking. The shift is a change in the ultimate unit of economic investigation from commodities ans individuals to transactions between individuals".

Economie et Institutions $-n^{\circ} 5-2^{e}$ semestre 2004 
plus que sur des objets, elle est de nature transactionnelle, on a pu parler ainsi à propos des approches de Dewey et Bentley ou de Commons d'"individualisme relationnel" [Emirbayer 1997 p.297] . Le connu et le connaissant ne peuvent être séparés, ne serait-ce que du fait de l'action de nommer qui est de nature relationnelle et réflexive (les mots ne prennent leurs sens que vis à vis d'autres mots). La connaissance est toujours contextuelle et dépend de l'intentionnalité de l'acteur. Il n'y a pas de connaissance "pure" indépendante du contexte (ou de la "situation"), de même qu'il n'y a pas d'objet "en soi" indépendant du sujet connaissant et de ses projets. L'action n'est donc pas déterminée uniquement par des préférences et des contraintes (ou incitations) mais également par un contexte (ou une "situation") et par l'interprétation de ce contexte. Les agents doivent attribuer un sens à leur environnement pour agir. La connaissance, dans un système social, est "subjectivement" dispersée du fait de cadre d'interprétation différents. La création et le maintien de systèmes d'action organisés suppose donc la création et le maintien de "dispositifs cognitifs collectifs", d'une "grammaire sociale", ou encore chez Commons de "règles d'action" (working rules).

Une telle perspective relationnelle ou transactionnelle permet d'appréhender la rationalité et la prise de décision par les agents de façon plus pertinente. En effet dans ce cadre, les fins et les moyens ne sont pas séparés mais se co-déterminent. Moyens et fins ne constituent jamais des données, ne serait-ce que parce que les acteurs doivent leur donner un sens. Le concept de "fin-visée" (endsin-view) de J.Dewey traduit cette interaction permanente des moyens et des fins. L'intentionalité (will de Commons) est inséparable de l'action et de la décision. L'incertitude est également intrinsèque dans cette perspective ${ }^{18}$.

\section{-Les "hypothèses comportementales"}

Ces éléments étant posés on peut prendre comme point de départ de l'analyse de Commons les hypothèses comportementales qui fondent son analyse et qui constituent un équivalent des hypothèses mobilisées dans l'approche néo-institutionnaliste (Williamson): la rationalité limitée et l'opportunisme, la première ayant la prééminence dans l'approche de Commons. Commons

18 Il faut souligner à ce propos la proximité des analyses de Knight avec le pragmatisme. Par rapport à ce que nous avons évoqué à propos de la notion de situation, il écrivait ainsi:"the essence of the situation is action according to opinion [...]"[1921, III.VII.5] ou encore "We perceive the world before we react to it, and we react not to what we perceive, but always to what we infer" [Ibid]. De plus: "It must be recognized[...] that no sharp distinction can be drawn between perception and reason"[1921, III.VII.9].

18 Economie et Institutions $-\mathrm{n}^{\circ} 5-2^{\mathrm{e}}$ semestre 2004 
reconnaît qu'une partie du comportement humain est calculatoire, raisonné, orienté vers un but, mais qu'en même temps, ce comportement implique des éléments d'ignorance, de stupidité, de passion [Commons 1934 p.390]. Dans ce cadre, comme l'affirme B.F.Kaufman [2002 p.8], les idées de base du concept de rationalité limitée se trouvent en germe chez Commons. Nous avons eu l'occasion de souligner que l'origine de cette considération se trouve dans la philosophie pragmatique, en particulier l'approche de J.Dewey auquel H.Simon a largement emprunté son concept de search (on trouve ainsi explicitement chez Dewey le concept de Quête, ou plus spécifiquement d'enquête) [Renault 1992]19. Commons introduit également la passion, l'émotion, en plus de la limitation de l'aptitude cognitive et de la limitation des informations [Kaufman 1999]. On sait que dans l'approche de Williamson ces éléments constituent des "frictions": cela est également le cas chez Commons [1934 p.100]. Dans son analyse du comportement et de la prise de décision, Commons met l'accent sur la notion d'incertitude via son concept de futurité (futurity). Pour Commons l'action est intentionnelle: toute action est orientée vers un but et donc tournée vers le futur. Echange et production sont donc orienté vers le futur et mettent en jeu les anticipations des agents; il s'agit donc pour les agents de prendre en compte un flux anticipé d'événements et pas seulement de mener à bien un calcul d'optimisation dans un monde statique. La prise en compte par Commons de l'action humaine est ainsi "volitionnelle" (volitional); comme l'écrit D.Hamilton [1953 p.63]: "Every individual was motivated by a purpose, and every action an individual took was a choice made from among perceived opportunities in order to advance that purpose. Economic activity in the aggregate was no more or no less than the continuous action of individuals making purposeful choices". L'approche institutionnaliste doit donc ainsi, selon Commons, prendre en considération la façon dont les individus se comportent dans une société humaine dont le futur est clairement reconnu comme étant imprévisible mais qui peut cependant être contrôlé partiellement par l'intermédiaire de l'action collective [Commons 1934 p.107]. Une autre ambition de l'approche institutionnaliste dans cette perspective est de comprendre comment se forment les anticipations des agents [Renault 1999], ce qui renvoie aux aspects institutionnels, nous y reviendrons.

L'opportunisme est également envisagé par Commons, la notion de conflit d'intérêt lié à la rareté et à la rivalité étant un principe de base de son analyse, il ne revêt cependant pas le caractère central qu'il possède dans l'analyse néo-institutionnaliste. Commons met ainsi en avant l'égoïsme (self-interest) qui est défini

19 Voir aussi J.Forest et C.Méhier [2001] 
comme "getting as much as possible and suffering little loss as possible(...) without regard to the effect on other people (...) unless (he) is restrained in some way that he cannot overcome" [1934 p.799]. Dans le cadre de son analyse des transactions, Commons met également en avant le fait que derrière la négociation se déroule un processus d'influence mutuelle, de "propagande", de persuasion...la transaction est en effet une unité de conflit, de dépendance mutuelle et d'ordre; les individus étant en rapport avec leurs semblables, le conflit des intérêts est inévitable [1934 p.57-58]. Le conflit des intérêts est cependant tempéré chez Commons. Il met en effet en avant le fait que la volonté humaine possède une faculté unique parmi les forces de la nature: celle de pouvoir limiter la puissance de son propre exercice. L'action collective en particulier a le rôle de contrôler l'action individuelle [1950 p.38], cela apparaît clairement dans le cadre des organisations.

D'autre part, Commons met en œuvre dans son analyse, si on suit la présentation de Kaufman [2002 p.9], des "fonctions d'utilité interdépendantes". Même si ce terme peut paraître inapproprié dans le cadre de l'approche de Commons, l'idée générale que cela implique est importante. Commons en effet, on l'a vu, admet l'égoïsme, l'opportunisme, les comportements de passagers clandestins..., cependant, selon lui, il est inapproprié de faire de ces motifs complémentaires des règles universelles. La confiance, la loyauté, l'amour, la honte, le désir de justice...apparaissent également comme des motifs d'action qui ont la caractéristique d'émerger par interaction, par communication [Renault 1999]. Les comportements coopératifs, l'attention portée à la justice...peuvent ainsi se révéler plus efficient que l'égoïsme et le calcul utilitariste pur dans les situations où les individus ont à travailler ensemble, en équipe, comme c'est le cas pour les firmes. Dans une large mesure cela renvoie au caractère "institutionnalisé" de l'esprit et au processus de socialisation qui l'a engendré.

Nous avons souligné ainsi que la volonté humaine pouvait suspendre sont propre exercice: "(...) this power of control may be expressed by the terms: performance, forbearance, avoidance" [Commons 1950 p.38]. Comme toujours chez Commons ces termes revêtent des acceptions subtiles et ont de multiples déclinaisons; on peut cependant essayer de préciser leur contenu:

-le terme "performance" traduit l'aspect dynamique et téléologique de la volonté, il signifie à la fois l'accomplissement d'une tâche ou d'une action et également le degré de réalisation de l'objectif,

20 Economie et Institutions $-\mathrm{n}^{\circ} 5-2^{\mathrm{e}}$ semestre 2004 
-le terme "forbearance" traduit la suspension temporaire et volontaire de l'action, la patience, l'attente imposée par les conditions, par le contexte,

-le terme "avoidance" traduit l'évitement d'une action, le non exercice de la puissance, de la volonté.

Selon Kaufman [2002 p.10], la notion de "forbearance" est particulièrement importante puisqu'elle renvoie au fait que les individus limitent de façon consciente l'exercice de leur pouvoir et donc les gains potentiels liés à sa mise en œuvre. C'est donc par l'intermédiaire de la "forbearance" que l'opportunisme n'est pas universel et cela même quand la probabilité de sanction est nulle. Kaufman ajoute un élément qui nous semble très important et qui rejoint notre analyse: "Forbearance arises from a shared emotional commitment to observe the rules of the game as a matter of ethical principle" (nous soulignons). Cela laisse la place pour un ordre raisonnable et négocié. D'une façon plus générale, c'est l'aspect institutionnel des organisations qui permet d'assurer la coordination des comportements et de limiter l'opportunisme.

\section{II-Coordination, action et dimensions institutionnelles des firmes}

\section{-Firme et connaissance dispersée}

Dans les tentatives récentes d'élaboration d'une approche autrichienne la firme apparaît car "elle permet d'accroître l'information contrôlée, structurée par les caractéristiques techniques de la production. Elle réduit aussi l'incertitude liée aux transactions marchandes et, en conséquence, les coûts liés à l'information qu'il faut rassembler pour effectuer des échanges" [Longuet 2001 p.37]. Une telle perspective semble assez proche, au moins dans sa problématique, de celle développée par l'économie des conventions [Ughetto 2000] pour laquelle la firme apparait comme un lieu de confrontation collective à l'incertitude dans une perspective de rentabilité. Selon O.Favereau [1989b p.90], la firme correspond alors à des "dispositifs cognitifs collectifs aménageant, au moyen de contrats et de contraints des interactions individuelles de nature à desserrer-par un processus d'apprentissage collectif-une contrainte globale de rentabilité minimale". La perspective autrichienne se confronte relativement peu cependant à cette contrainte de rentabilité de façon explicite, hormis via la sélection du marché. On peut cependant considérer qu'une telle perspective n'est pas absente par l'intermédiaire du rôle de l'entrepreneur en tant que chercheur de rente ou de profit. 
L'incertitude et les limitations cognitives apparaissent donc comme des explications originelles de l'existence de firmes, celles-ci ne se distinguant des autres formes d'organisation que par leur nature ou par leur objet. Un autre élément usuel dans la théorie de la firme est la questions des limites de la firme, correspondant à la question posée par Coase de savoir pourquoi il n'y a pas une seule grande firme. Ici encore, sans entrer dans les détails, on peut en simplifiant référer cette question à l'arbitrage entre centralisation et décentralisation, dont l'appréhension s'enracine en dernière analyse dans le débat des années 1920-1930 sur le calcul socialiste [Klein 1996].

Dans une firme en effet, un arbitrage entre centralisation et décentralisation des décisions doit être fait, arbitrage qui doit également être articulé avec la qualification des règles issue de l'approche hayékienne [Garrouste 1999 p.890]: abstraites ou concrètes, générales ou spécifiques, tacites ou codifiées. On peut rappeler de façon simple à ce propos que l'approche autrichienne repose sur une distinction entre institutions pragmatiques et organiques d'une part et ordres spontanés et délibérés (ou planifié) d'autre part. La première typologie est due à C.Menger (1883): ainsi, alors que les institutions pragmatiques sont le produit de "causes socialement téléologiques", les institutions organiques sont le produit inintentionnel des actions d'innombrables sujets économiques poursuivant leur propre intérêt. L'approche de Menger est essentiellement destinée à fournir une explication de l'émergence des institutions. La seconde typologie due à F.A.Hayek (1973) complète celle de Menger et sa distinction est fondée sur les différentes règles d'organisation qui sous-tendent les deux types d'ordres. Les règles qui sous-tendent l'ordre spontané sont abstraites, générales et non téléologiques, alors que les règles qui sous tendent les ordres délibérés sont construites, spécifiques et concrètes.

Comme le soulignait P.G.Klein [1996 p.4]: "All organizations plan, and all organizations, public and private, perform economic calculation. In this sense, the calculation problem is much more general than has usually been realized". Ainsi, chaque agent à l'intérieur d'une firme est susceptible de posséder une connaissance spécifique du fait de la division du travail; si la centralisation de cette connaissance est impossible, la firme aura intérêt à décentraliser ${ }^{20}$.

20 Hayek a mis en avant l'importance de la coopération des salariés dans le cadre des processus d'apprentissage. Comme il l'écrit dans "L'utilisation de la connaissance dans la société" (1945): "Chaque individu possède un savoir unique [...] dont l'usage ne peut être fait que si on laisse à cet agent le soin de

22 Economie et Institutions $-\mathrm{n}^{\circ} 5-2^{\mathrm{e}}$ semestre 2004 
La coordination risque alors de devenir plus problématique du fait de la compatibilité des plans d'action qui ne va plus de soi dans ce cas. La cohérence décisionnelle impliquerait donc l'instauration d'un système de communication assurant un certain degré de planification. Un tel système a évidemment un coût qui sera croissant avec le degré de décentralisation de la firme [Longuet 2001 p.38]. Ainsi: "c'est l'existence de ce coût qui réduit le domaine de la planification consciente et qui constitue le pendant aux coûts de transaction" [Ibid.].

Il faut voir également que la dialectique centralisation/ décentralisation repose sur les limites cognitives des dirigeants de la firme. Il faut donc ajouter autre chose aux "coûts d'information": en effet la centralisation des informations est dans une large mesure impossible. Dans la perspective hayékienne, l'intégration ou la soumission des interactions spontanées à un commandement, à une hiérarchie ou une planification risque de perturber les plans individuels et de conduire à l'inefficacité. La firme représentant à la fois un ordre délibéré et un ordre spontané [Garrouste 1999] est confrontée à ce type de dilemme. L'intervention sur les ordres spontanés en déclenchant un processus auto-entretenu de centralisation va rencontrer les limites cognitives du planificateur: on risque d'aboutir à des choix incohérents, inefficaces ou inadaptés. D'un autre côté, le développement "anarchique" des ordres spontanés internes risque d'aller à l'encontre de la notion de but commun, central dans l'existence d'une firme: il y a alors risque de décomposition ou d'éclatement.

\section{-Les constructions mentales partagées}

Un élément très important est alors mis en avant à juste titre par S.Longuet [2001] à la suite de Witt [1998] pour assurer malgré ce dilemme la cohérence de l'organisation, c'est à ce stade que les analyses autrichiennes rejoignent la perspective institutionnalistepragmatique et c'est essentiellement cela que nous développerons. La coordination des plans et des actions dépendra largement des "constructions mentales partagées" qui facilitent la convergence des anticipations et des comportements ${ }^{21}$. Il s'agit là nous semble t-il d'une thématique très importante et nouvelle par rapport au cadre usuel de l'économie de la firme et des organisations et qui rencontre aujourd'hui de nombreux échos, par exemple dans le cadre des approches "conventionnalistes" ou évolutionnistes, mais aussi, nous

prendre lui-même les décisions ou si ces décisions sont prises avec sa coopération active", cité par Bensaïd et Richebé [2001 p.79]

21 On retrouve ainsi les "mondes communs" évoqués par Salais et Storper [1993] ou la perspective de Boltanski et Thévenot [Renault 1997].

Economie et Institutions $-n^{\circ} 5-2^{e}$ semestre 2004 
y reviendrons dans le cadre de l'approche institutionnaliste. L'origine de ces considérations en terme de "modèles mentaux partagés" ou de "constructions mentales partagées" doit être recherchée dans les analyses psychologiques initiées par Hayek dans "The sensory order" [1952] comme nous l'avons déjà évoqué. Comme l'écrit P.Garrouste [1999 p.888], c'est dans cet ouvrage que s'enracine le subjectivisme hayékien. Les individus mettent en effet en place des systèmes de catégorisation et de classification du monde communs, ce qui permet la compréhension. Cependant, la construction de tels systèmes n'est pas consciente ou délibérée et la confrontation à l'environnement des individu est spécifique, ce qui implique que les individus peuvent avoir des perceptions différenciées: "Ce phénomène justifie que les individus, qui deviennent alors des sujets, aient un ensemble de connaissances qu'ils ne peuvent toutes transmettre, premièrement parce que, pour certaines d'entre elles, ils n'ont pas conscience de les posséder, deuxièmement, du fait de l'absence de signification (nous soulignons) qu'elles sont susceptible d'avoir pour les autres" [Garrouste 1999 p.888]. On comprend dans ce cadre l'enjeu de la définition de "mondes communs" (ou de routines) pour les firmes, ce qui renvoie à leurs dimensions institutionnelles.

Compte tenu du caractère fragmenté et dispersé de la connaissance, les "mondes communs" ne peuvent qu'avoir un degré de généralité élevé, ce qui permet également de faire face à des événement émergents [Foss 1998b p.20]. Quand il s'agit d'appréhender ces aspects on trouve de façon récurrente dans la littérature autrichienne la référence à l'approche de A.Schütz. En effet, dans un environnement factuel et comportemental caractérisé par une incertitude radicale, la coordination doit faire face à l'émergence de la nouveauté. A.Schütz distinguait dans les événements des caractéristiques typiques et uniques. Le processus de "typification", déjà évoqué, apparaît ainsi comme un aspect de la façon dont les agents et les firmes perçoivent leur environnement. Les aspects typiques matérialisent les aspects stables de l'environnement qui peuvent être perçu et/ou anticipés, et qui favorisent donc la coordination entre les agents (ils peuvent constituer l'équivalent de "points focaux"). Les aspects uniques, au contraires, ne sont pas répétés et sont liés à des circonstances spatiales, factuelles et temporelles spécifiques [Foss 1998b p.20-21; Longuet 2001 p.40]. La plupart des événements représentent un mélange des deux aspects, typiques et uniques, qui permettent de faire face à l'émergence de la nouveauté, de la rendre intelligible. On comprend alors comme le souligne N.J.Foss [1998b p.20] que "(...) One may think of the act of choosing which coordination game to play, of making sense out of new events (and communicating this) etc...as more fundamental than the problem of inducing agents to make

24 Economie et Institutions $-\mathrm{n}^{\circ} 5-2^{\mathrm{e}}$ semestre 2004 
specific actions within an already well understood decision situation". Ainsi, dans une perspective autrichienne, le management peut être conçu comme permettant de résoudre (ou de contribuer à résoudre) des problèmes de coordination de niveau inférieur (concrets) alors que la planification (ou plus exactement le leadership) constituerait une activité de niveau plus élevé en sélectionnant des "précédents" ou des "points focaux" ayant un degré de généralité plus élevé et relevant du caractère typique des événements. Dans ce cadre, relativement au marché, la firme peut présenter un avantage via le recours au mécanisme de planification et de leadership pour résoudre les problèmes de coordination. Le marché ne peut en effet procéder que par un mécanisme d'essais et d'erreur qui peut se révéler plus coûteux [Foss 1998b p.20]. Comme le souligne S.Longuet [2001, p.40], une telle conception permet de référer la coordination au sein de la firme à la création d'un "environnement communicationnel commun", ce qui renvoie aux travaux de T.Fu-Lai Yu [1999] et de Schütz: "Dans cette perspective, la firme peut permettre une coordination interne en facilitant la typification qui garantira une interprétation commune (nous soulignons) de certains phénomènes, interprétation qui servira de base aux anticipations". Dans ce cadre, la répétition des activités de production ou d'administration joue un rôle, mais les règles sont évidemment également essentielles en donnant un cadre pour l'action et en favorisant l'utilisation des connaissances particulières par les agents dans un environnement commun. A.Schütz, dans sa définition des typifications se référait ainsi à la notion de règle, en effet les typifications sont: "[...] a set of rules and recipes for coming to terms with typical problems by applying typical means for achieving typical ends" [1967 p.27-nous soulignons].

La question des règles apparaît ainsi particulièrement importante pour faire face aux problèmes de coordination liés à la dispersion des connaissances. Il serait trop long de revenir ici sur la totalité de l'analyse des règles développée par le courant autrichien, on pourra à ce propos consulter les travaux de P.Garrouste [1999, 2002], P.Dulbecco et P.Garrouste [2000], M.Bensaïd et N.Richebé [2001] sans être exhaustif. Pour notre propos on peut, pour simplifier, affirmer que tout système organisé repose sur des règles (règles de comportement en particulier) qui sont à la fois, comme nous l'avons déjà évoqué, volontairement crées et spontanées, explicites et tacites, concrètes et abstraites, et qui ont fatalement des effets non négligeables sur les cadres cognitifs des individus. Par rapport au problème général de la coordination, la coordination organisationnelle présente un aspect volontaire, orienté vers un but, qui est plus marqué. L'entrepreneur trouve alors logiquement sa place dans l'approche autrichienne [Witt 1998], ce qui n'est pas 
forcément le cas pour d'autres approches. Ainsi, pour S.Longuet [2001 p.41], le concept d'entrepreneur peut être référé aux problèmes cognitifs émergeant au sein de la firme.

Si on conçoit en suivant Fu-Lai Yu [1999] la firme comme une solution, au moins partielle, à des échecs de coordination, cela signifie que les "typifications", ou les modèles qui guidaient le comportement des agents ont échoué pour garantir le succès et la compatibilité des plans. Dans ce cadre: "Le fondateur de la firme, en mettant en place des lignes d'autorité et de communication dont l'objectif explicite est de favoriser l'atteinte de buts communs, constitue un contexte qui permet de subordonner les objectifs individuels à l'objectif commun. Les actions prennent ainsi une dimension typique perceptible par chacun" [Longuet 2001 p.41]. L'entrepreneur en créant une firme construit un "monde cohérent de connaissance et une communauté culturelle (nous soulignons)" qui fonde "la compréhension et le consentement mutuel". On rejoint alors la perspective de nombreux travaux, en particulier ceux de U.Witt [1998, 2000], mais également nous semble t-il ceux des "constitutionnalistes" [Vanberg 1998]. Cet aspect institutionnel des firmes renvoie également aux travaux de L.Lachmann pour lequel: "An institution provides a means of orientation to a large number of actors. It enables them to coordinate their actions by means of orientation to a common signpost" [Lachmann 1970 p.49]. Dans ce cadre et en adoptant le langage de Schütz une institution correspond à un mode-d'action-typique-partagé (shared course-of-action-type) [Foss 1996 p.81]. Il faut voir également que cela trouve un écho dans la notion d'"attitude" chez G.H.Mead, puisqu'une attitude est définie comme une séquence de comportement internalisée organisée (organized internalized behavior sequence) ${ }^{22}$. Il faut voir en ce sens que toute institution apparaît comme le résultat des efforts de coordination des individus qui tentent d'interpréter les événements externes et de donner un sens aux interactions sociales et économiques [Fu-Lai Yu 2003b p.249], en ce sens dans la perspective de Schütz qui rejoint celle développée par G.H.Mead, la succession des interaction de "la vie de tous les jours" (everyday life) amène les individus à construire la catégorie de 1'"autre" et ainsi à pouvoir donner un sens aux actions des autres, permettant la coordination ${ }^{23}$.

Dans cette perspective, l'approche autrichienne, et celle de Hayek en particulier, semble adaptée pour prendre en compte

22 Voir:T.J.Scheff 1993

23 Comme nous l'avons souligné à propos de Mead et de la sociologie interactionniste, cette perspective est issue de la théorie des sentiments moraux de Smith. Voir Hörwitz [2001]

26 Economie et Institutions $-n^{\circ} 5-2^{e}$ semestre 2004 
l'émergence de nouvelles formes organisationnelles dans le cadre de ce qu'il est convenu d'appeler une "économie fondée sur la connaissance", où la connaissance apparaît comme de plus en plus dispersée. La communauté, qui apparaît comme une structure informelle basée sur un échange coopératif volontaire, des rapports de confiance, une adhésion "spontanée" à des objectifs communs, permet de développer et de prendre en charge des processus d'apprentissage qui, par nature, sont sociaux. On retrouve alors l'idée que la communauté repose sur l'existence d'une proximité cognitive et organisationnelle, d'une connaissance commune, d'un langage commun, de modèles mentaux partagés. Elle permet ainsi à la fois la coordination des connaissances dispersées et l'émergence de la nouveauté, la production de nouvelles connaissances [Diani 2002 , 2004]. On pourrait alors considérer la firme comme "une myriade de communautés interconnectées et complémentaires dans l'accumulation et la génération de la connaissance" [Diani 2002 p.7]. Cette perspective s'articule avec celle de U.Witt [1998 p.166] pour lequel: "Cognitive commonalities, that is, socially shared tacit knowledge including knowledge about social models of behavior, may emerge spontaneously from intense communication as an unintended collective outcome and may, as such, be difficult to influence. Sometimes, however, the institutional set up of the interactions assigns certain individuals a position in which they get a chance to shape the communication processes and thus to exert an influence on the collective outcome. The firm organization is a case in point (nous soulignons)"

Il est évident qu'une telle conception ne peut permettre de constituer une théorie "complète" de la firme, au moins de la façon dont nous avons présenté les choses. Il faut également souligner que de nombreux aspects pertinents de l'approche autrichienne n'ont pas été, volontairement, développés; en particulier ceux liés aux aspects productifs des firmes. Il faudrait en effet à côté des aspects cognitifs liés à la coordination que nous avons privilégiés ajouter des aspects liés à la structure temporelle de la production [Dulbecco et Garrouste 2000, Yu 1999, Longuet 2001] et aux "capacités" (capabilities) et compétences [Foss 1998a et 1998b, Ioannidès 1999]. Il nous semble cependant que les aspects cognitifs et communicationnels sont particulièrement pertinents et permettent d'établir un liens avec l'approche institutionnaliste qui se place dans une perspective comparable à celle des autrichiens concernant la question de l'action organisée et de la coordination. Il ne faut cependant pas oublier que l'apparent syncrétisme que nous semblons mettre en avant cache des différences philosophiques, épistémologiques et méthodologiques. En particulier on doit souligner que l'approche autrichienne demeure largement dualiste (ordre spontané/ordre 
délibéré) alors que l'approche institutionnaliste refuse ce dualisme [voir Renault 1992 et Chavance 2001]. D'autre part il faut bien reconnaître, et cette critique est aussi partiellement valable pour certains aspects de l'approche institutionnaliste, que cette approche demeure largement conceptuelle et apparaît encore relativement pauvre d'un point de vue de son opérationalisation. Un autre point critique est le caractère relativement désincarné de l'organisation: excepté via l'entrepreneur ou le leadership, il n'y a pas de référence réelle eu pouvoir et à la négociation autour des règles dans l'approche autrichienne. Ainsi, excepté via la dialectique centralisation/décentralisation l'organisation "interne" est d'une façon générale peu explorée par l'analyse autrichienne.

\section{-Institution et action collective}

L'analyse de Commons constitue avant tout une analyse de l'action collective s'inscrivant dans une problématique institutionnaliste. Institution et action collective sont donc les deux éléments centraux, mutuellement interdépendants, qui fondent l'analyse. On peut rappeler brièvement les définitions que Commons [1931] donne de ces deux termes:

-"(...) We may define an institution as collective action in control, liberation and expansion of collective action"

-"Collective action ranges all the way from unorganized custom to the many going concerns, such as the family, the corporation, the trade association, the trade union, the reserve system, the state. The principle common to all of them is greater or less control, liberation and expansion of individual action by collective action."

Ces deux définitions montrent comme le soulignent L.Bazzoli et V.Dutraive [1998b p.1], que l'on ne peut séparer institution et organisation, il s'agit de deux faces d'un même phénomène. Le concept d'action collective organisée apparait donc en ce sens particulièrement important la firme constituant une forme particulière d'action collective organisée. Le terme "going concern" est ainsi utilisé par Commons: "to denote an association of individuals who together engage in ongoing coordinated activity directed to ends foreseen in the future" [Ramstad 1994 p.95]. La hierarchie des définitions suggère également que l'aspect institutionnel a le primat sur l'aspect organisationnel puisque les phénomènes institutionnels "(...) définissent la nature, les conditions de fonctionnement et les modalités d'évolution des organisations (...)" [Bazzoli et Dutraive

28 Economie et Institutions $-n^{\circ} 5-2^{e}$ semestre 2004 
1998 b.2]. Il nous semble que c'est le cas pour des raisons cognitives et comportementales.

Toute théorie des organisations repose en effet, explicitement ou implicitement, sur des hypothèses comportementales, c'est le cas pour la théorie de l'agence, la théorie des contrats incomplets ou encore le néo-institutionnalisme. A cet égard, rationalité limitée et opportunisme apparaissent comme des éléments saillants. Nous avons essayé de montrer que dans le cadre de l'analyse autrichienne, la dépendance entre la théorie de l'action et la théorie de la firme était forte. C'est également le cas chez Commons. Si on veut simplifier les choses, on peut considérer que l'analyse de Commons prend d'abord en compte la rareté constitutive qui conduit inéluctablement à la rivalité entre les individus. Le problème de l'ordre social apparaît alors évidemment comme fondamental. Commons observe ainsi que les individus sont confrontés à trois défis pour surmonter la rareté et ses effets: la résolution des conflits, la prise en compte de l'interdépendance et la création d'un ordre civique [Kaufman 2002 p.2]. On peut donc affirmer d'une certaine façon que l'action collective, et ses différentes formes organisées, apparaît comme un moyen de faire face à la rareté et à ses conséquences. On peut noter également que chez Commons la rareté peut être effective, actuelle, ou anticipée, ce qui met l'accent sur l'incertitude intrinsèque. La souveraineté (le pouvoir) apparaît également dans ce cadre comme un point important de l'analyse de Commons puisque la souveraineté, qui s'exerce à différents niveaux, permet, au moins partiellement, de prendre en compte l'ordre, la dépendance et le conflit ${ }^{24}$. La souveraineté opère par l'intermédiaire de règles d'action (working rules) qui, elles aussi, sont de nature diverse selon le niveau auquel la souveraineté opère. Comme le souligne B.F.Kaufman, ces règles d'action sous tendent ce que williamson appele des structures de gouvernance. Ainsi: "In addition to maintaining order and resolving conflicts, the sovereign power must also use working rules to foster cooperation in production and exchange and to resolve the numerous problems created by the pursuit of self-interest in an interdependant world. These functions do not arise automatically from a Smithian system of "natural liberty" but have to be induced by appropriate incentives created by the visible hand of sovereignty and enforced by various sanctions including the threats of physical violence" [Kaufman 2002 p.3]. Cet aspect de

${ }^{24}$ L'autorité, ou plus généralement le pouvoir, permettent de coordonner les comportements individuels par l'intermédiaire de règles formelles ou informelles partagées assurant la stabilité des anticipations. Le "leadership" n'est donc pas simplement cognitif mais est également supporté par un système de sanction de nature diverse. 
pouvoir, intrinsèque à l'action collective, était peu présent dans l'approche autrichienne. Nous avons dit précedemment que l'action collective exhibait un continuum de formes, de la moins organisée à la plus organisée; la souveraineté possède la même caractéristique continuiste: toute action organisée implique un certain degré de souveraineté et crée et met en œuvre ses propres règles d'action. Pour Commons il existe ainsi une "hiérarchie de gouvernements": "(...) such as business firms, labor organizations, churches and families" [Commons 1950 p. 74]. Tout cela implique donc qu'au lieu d'individus isolés nous avons de multiples hiérarchies qui régulent le comportement individuel.

\section{-Organisation et règles d'action}

Dans ce cadre la conception de Commons d'un comportement individuel marqué par un concernement pour les autres, similaire à l'analyse de Mead (other regarding behavior) n'est pas neutre pour la conception économique de l'organisation et renvoie de fait à l'importance et au rôle des normes sociales dans la détermination du comportement, à l'aspect institutionnel des organisations ${ }^{25}$. Comme le souligne Kaufman [2002 p.10]: "Norms, in effect, helps create "selfenforcing" contracts. This conclusion is the fundamental point argued in Commons's book "Industrial goodwill" (1919)-that creating mutual feelings of reciprocity and fair treatment ("goodwill") allow organisations to escape inefficient prisonner dilemma outcomes, and is now routinely cited in modern day management texts as a basic principle for effective organizational performance". Dans ce cadre on sait que Williamson met en avant les incitations "fortes" du marché, ce qui avantagerait ce type de structure de gouvernance. Cette proposition apparaît incomplète dans la perspective de Commons. En effet, les organisations fournissent également des incitations fortes, simplement elles font appel à d'autres émotions ou sentiments. Commons met ainsi en avant l'esprit de corps ou d'équipe: "Team spirit not only subordinates individual self-interest to the good of the group but also arouses (motivates) people to high levels of performance-quite possibly to a higher level than can the prospect of pecuniary self-gain" [Kaufman 2002 p.10]. La référence mobilisée récemment par la littérature économique à la "culture" à des valeurs partagées ou aux dimensions "communautaires" des firmes renvoie à cette perspective.

25 Ici encore il faut souligner la proximité des conceptions de Commons avec celle des autrichiens et en particulier Hayek, proximité qui s'enracine selon nous dans les aspects cognitifs de leurs théories de l'action [Voir C.G.Leathers 1989]

30 Economie et Institutions $-\mathrm{n}^{\circ} 5-2^{\mathrm{e}}$ semestre 2004 
Dans l'approche de Commons les normes, les règles, les habitudes...issues de l'action collective, de l'interaction entre les individus, constituent des institutions qui permettent la compatibilité des comportements des individus. L'action individuelle est donc déterminée par les institutions. Les institutions les plus codifiées et orientées vers un but constituent des "going concerns" [Dutraive 1993 p.92], ce qui se rapproche de la conception autrichienne telle que nous avons pu l'exposer, en particulier à travers la notion d'institution pragmatique (ou organisation). Contrairement à l'approche de Williamson, il n'y a pas dans ce cadre dichotomie entre organisation (ou institution) et marché; les institutions ne résultent pas de l'imperfection du marché, elles sont au contraire coextensives de l'action humaine, de la plus inorganisée aux formes les plus élaborées. Les individus qui échangent sur le marché sont en effet des individus socialisés, ce qui est traduit chez Commons par la notion d'esprits institutionnalisés (institutionalized mind) [Renault 1999].

Le concept de Commons aujourd'hui le plus communément répandu est celui de transaction, c'est d'ailleurs pour ce concept que Williamson le crédite. Cependant le concept de transaction de Commons est notablement plus complexe que le concept mis en œuvre dans l'analyse néo-institutionnaliste. En effet chez Williamson, la transaction est définie comme une opération consistant à transférer des biens et services via une interface technologiquement séparable. L'appréhension de la notion de transaction pour Commons prend sa source dans sa conception "volitionnelle" (volitional) de l'action. L'échange n'apparaît plus ainsi comme une simple transmission, mais reflète une négociation, un processus, qui aboutit à un transfert de droits de propriété. La substitution de la notion de transaction à la notion d'échange permet d'introduire deux éléments:

-le temps: le terme transaction retrace le développement temporel de l'action effective, y compris les anticipations mutuelles, alors que le terme échange renvoie à un instant du temps.

-la superstructure institutionnelle: c'est elle en effet qui permet à la fois le transfert de propriété sous le contrôle de la loi et l'établissement d'un compromis, d'un accord dans l'action résultant de processus de négociation [Commons 1950 p.46].

On doit ici souligner la double nature de la notion de transaction: une "nature matérielle" (un transfert de droits de 
propriété) et une nature "cognitive" (via le processus de négociation qui met en scène les anticipations mutuelles des agents, leurs modèles mentaux, leur connaissance).

Les agents agissent ainsi au sein de Going concerns, qui sont caractérisés par des règles d'action spécifiques (working rules) qui représentent des schémas d'action usuels partagés par les membres du groupe (ces règles pouvant être formelles et/ou informelles). Les règles d'action, et les actions des individus les actualisant, révèlent la volonté collective et l'objectif social de l'organisation. Si un individu participe successivement à plusieurs "going concerns", il devra à chaque fois intégrer les règles d'action; au cours de ce processus d'apprentissage, de socialisation, l'éthique et les objectifs de l'individu en viennent à refléter ceux du "going concern" auquel il participe, l'esprit est peu à peu "institutionnalisé"

Les organisations (going concerns) sont donc conçues pour supporter les transactions. Dans ce cadre, l'aspect cognitif des transactions est très largement souligné par Commons. Nous avons développé ce point [Renault 1997, 1999] en montrant la relation de l'approche de Commons avec celle de Peirce, Dewey et Mead, en soulignant le caractère communicationnel de cette approche [voir également Albert et Ramstad 1997, 1999]. Cela correspond bien à l'approche transactionnelle définie par Dewey et Bentley.

\section{-Coordination des comportements et routines}

La coordination des comportements individuels par l'intermédiaire de l'aspect institutionnel des organisations est appréhendée par Commons. Il s'agit ainsi de "(...) découvrir-ou plutôt aider à créer- une structure interpersonnellement partagée de transactions. De la même façon que la conversation ne peut prendre place sans structures de significations partagées, la transaction ne peut avoir lieu dans un vide institutionnel"[Langlois et Cosgel 1998 p.112]. L'analyse de Commons, en particulier à travers la "psychologie négociationnelle" met en jeu la communication et l'intersubjectivité. Les cadres communs de pensée et d'action permettent la coordination; les institutions représentent des réactions communes de tous les membres d'une collectivité à une situation particulière. G.H.Mead, dont la conception est très proche de celle de Commons, cite alors les droits de propriété comme exemple: "Les institutions de la société sont ainsi des formes d'activité sociale organisées de telle sorte que les membres individuels de la société peuvent agir de la manière requise en adoptant l'attitude des autres à l'égard de ces activités" [Mead 1963 p.222]. La capacité de se mettre à la place des autres, l'empathie (la sympathie au sens de

32 Economie et Institutions $-n^{\circ} 5-2^{e}$ semestre 2004 
Mead), l'identification, apparaissent très important et cela se retrouve chez Commons, en particulier à travers son concept d'esprit institutionnalisé, il nous semble que cette perspective est complémentaire de celle de Schütz et des autrichiens évoquée précédemment. Commons soulignait ainsi que la coutume intervient en tant qu'"opinion collective contrôlant l'opinion individuelle" et ces opinions sont "les représentations auxquelles les habitudes de l'individu doivent se conformer si les individus ont à travailler ensemble" [Commons 1934 p.698-notre traduction]. Les coutumes définissent un cadre, un "champ d'opportunité" qui permet aux anticipations de trouver un point fixe. Dans le cadre des transactions, chacun cherche en effet à réduire l'incertitude concernant les actions potentielles des autres [Biddle 1990 b p.3]. Chez Commons, les représentations communes, les normes, les institutions...permettent la formation pour l'individu de ce que J.Habermas appelle une "attente de comportement généralisé" [Habermas 1987b p.422] qui revêt deux sens: un sens normatif (les individus sont en droit d'attendre quelque chose, du fait par exemple de l'existence de règles légales, d'un contrat...) et un sens cognitif (un événement, une action, sont "pronostiqués" ou anticipés).

Ce cadre cognitif peut s'appliquer comme l'a montré récemment E.Lorenz [2000, 2002] à l'analyse des "routines" dans les organisations. On connaît l'importance que les routines, et leur évolution, ont pris depuis l'ouvrage de Nelson et Winter (1982) et le développement de l'approche évolutionniste de la firme. Ces développements ont largement mis l'accent sur les aspects cognitifs des routines, dans une perspective assez largement individualiste, et ont négligé leur aspect "politique". Or cet aspect, que nous avions souligné [Renault 1999 p.288], est mis en avant par Lorenz pour souligner la pertinence de l'approche de Commons. En effet, dans l'approche de Commons, l'opinion, les représentations et l'action, ne peuvent être séparés, l'action est "l'opinion-en-action" et elle est gouvernée par des "hypothèses habituelles" et coutumières. De cette façon: "(...)social sciences investigates habitual and customary assumptions as an explanation of transactions (nous soulignons)" [1934 p.698]. les coutumes sont apprises par les individus et deviennent progressivement inconscientes, elles deviennent ainsi des "routines":

"When a new worker goes into a factory or a farm, or when a beginner starts in a profession or business, everything may be novel and unexpected because not previously met in his experience. Gradually he learns the ways of doing things that are expected from him. They become familiar. He forgets that they were novel when he began. He is unable even to explain them to outsiders. They have 
become routine taken for granted. His mind is no longer called upon to think about them (...). We speak of such minds as institutionalized. But all minds are institutionalized by whatever habitual assumptions they have acquired and they take for granted, so that they pay no attention to them, except when some limiting factor ${ }^{26}$ emerges and goes contrary to what they were habitually expecting (nous soulignons)" [1934 p.697-698].

Dans ce cadre, la réduction de l'incertitude et la sécurité des anticipations sont atteintes par l'intermédiaire des coutumes ou des règles d'action. Comme nous l'avons souligné, ces régularités comportementales sont enracinées dans les croyances ou hypothèses des individus au sujet du comportement probable des autres dans des situations d'interaction [Lorenz 2000], et donc dans l'apprentissage que les individus font en tant que membres d'une collectivité, d'un going concern. Dans ce cadre on peut rapprocher ici encore les "hypothèses habituelles" de Commons et les "Typifications" de Schütz.

Comme le fait remarquer E.Lorenz, un autre apport de Commons concerne la distinction entre habitude et coutume qui permet de prendre en compte le fait que le comportement individuel est toujours ancré dans un contexte social et que les coutumes présentent toujours un aspect socialement contraignant [Lorenz 2000 p.196-198]. Pour simplifier on peut dire que les habitudes matérialisent un comportement répété au niveau individuel ou local alors que les coutumes matérialisent la conformité de l'individu à certaines règles de comportement largement diffusées et partagées. Les coutumes présentent donc un degré de généralité plus élevé. Nous avons souligné que cette distinction, issue de l'approche de C.S.Peirce et de la philosophie pragmatique [Renault 1992], se retrouve sous une forme qui nous semble encore très pertinente chez G.H.Mead [Renault 1999]. Mead établi en effet une distinction entre la convention, par essence partielle et informelle, largement tacite, et l'institution, plus générale, matérialisant des réactions communes et qui ont le caractère plus codifié de "grammaire sociale". Pour Mead: "les conventions sont des réactions sociales isolées qui ne constituent pas la nature de la communauté dans son caractère essentiel (...)" [Mead 1963 p.223]. Dans l'approche de Mead, comme dans celle de Commons, la pression collective à l'action conforme permet d'orienter l'apprentissage individuel dans des directions socialement acceptables.

26 Notons d'ores et déjà que cela induit l'agent à mettre en oeuvre un processus d'enquête au sens de Dewey et à une révision des hypothèses habituelles, ce que nous développerons dans la partie III

34 Economie et Institutions $-\mathrm{n}^{\circ} 5-2^{\mathrm{e}}$ semestre 2004 
Partant d'une conception originale de l'action, les approches autrichiennes et pragmatiques-institutionnalistes mettent donc en évidence l'importance des cadres communs de pensée et d'action, des règles, dans la coordination des comportements. Leur approche permet également d'appréhender de façon pertinente l'innovation, la création.

\section{III-Création, innovation et entrepreunariat}

\section{-La vigilance entrepreneuriale}

Dans une large mesure, les approches autrichiennes appréhendent les phénomènes de création, d'innovation et de développement à travers l'entrepreneuriat (entrepreneurship). Il nous semble que ce qui est essentiel dans ce cadre correspond aux éléments développés précédemment concernant les fondements cognitifs de l'action. U.Witt [2000 p.734] souligne ainsi l'importance d'un fondement comportemental pour une approche dynamique de la firme. De la même façon, la vigilance entrepreneuriale et la découverte/création de nouvelles opportunités par l'entrepreneur évoquées par I.Kirzner trouvent des fondements cognitifs et comportementaux pertinents dans le cadre d'une théorie renouvelée de la firme.

Ainsi, si on reprend la définition donnée par I.Kirzner [1997 p.72] de la "vigilance entrepreneuriale" (entrepreneurial alerness), c'est à dire: "[...] an attitude of receptiveness to available but hitherto overlooked opportunities", elle doit être mise en parallèle avec le fait également souligné par Kirzner que les choses n'existent pas si les individus ne les connaissent pas ou ne les perçoivent pas. Ces éléments renvoient aux cadres cognitifs et interprétatifs qui soustendent le comportement humain. Ainsi T.Fu-Lai Yu [2001 p.47] peut affirmer que le processus de découverte entrepreneurial est associé au cadre d'interprétation des individus, au stock de connaissance disponible, dérivé de l'expérience quotidienne des individus, comme c'est le cas dans l'approche de A.Schütz. En particulier, la découverte signifie que l'acteur interprète l'information d'une façon différente de ce que perçoivent les autres individus. En effet comme nous l'avons déjà évoqué, dans la perspective de Schütz, les individus à tout moment du temps possèdent un stock de connaissance disponible qui leur permet d'interpréter leurs expériences passées et présentes et détermine leurs anticipations du futur [Fu-Lai Yu 2001 p.53]. Cependant, les individus font face à des événements ou des informations nouvelles qu'ils peuvent avoir du mal à interpréter, 
auxquels ils ont des difficulté d'attribution de sens. Le cadre d'interprétation et la connaissance disponible étant pris en défaut, la nouveauté pourra être rejetée comme une déviance ou un obstacle ou simplement ignorée. En ce sens l'entrepreneur sera capable au contraire de modifier son cadre d'interprétation et sa connaissance pour donner sens au nouveau ${ }^{27}$. Cela correspond également à la théorie de l'esprit de Hayek pour lequel les systèmes de classification et de catégorisation sont sans cesse confronté à la nouveauté et soumis à une dynamique d'évolution en établissant de nouvelles relations, ou de nouveaux systèmes de relations, ce qui correspond à la perspective relationnelle ou transactionnelle. Ainsi: "Creative activity thus involve the shifting together of different reference frames that would usually be oredered differently and be seen as incompatible until something clicks into place as a new way of looking at how things fit together. Such discoveries means that the actor escapes from the existing patterns of interpretation and reorganises ideas in new sequences" [Fu-Lai Yu 2001 p.54-55].

Dans ce cadre, l'imagination, la capacité d'élaborer des "fantaisies" (pour reprendre le terme de Schütz), apparaissent comme particulièrement importantes, ce qui est également mis en évidence par B.J.Loasby [2001]. Cette notion de "fantaisie" se réfère à la capacité de construction de monde possédée par l'esprit humain [FuLai Yu 2003 p.400]. Ces "fantaisies" vont d'un imaginaire pur sans lien direct avec la réalité expérimentée à des plans d'action plus réalistes qui concernent l'activité entrepreneuriale ${ }^{28}$. D'une certaine façon ces "fantaisies" entrepreneuriales revêtent une dimension sociale puisque la construction mentale d'un plan ou d'une conception entrepreneuriale doit prendre en compte le milieu social, par exemple les clients potentiels [Ibid.]. Ces "fantaisies" se confrontent à la réalité sous la forme de problème de cohérence pratique, tel par exemple la croyance des acteurs dans la possibilité de transformer leur projet en réalité [Schütz et Luckmann 1989 p.2427]. Ces dimensions de cohérence pratique reposent sur la considération d'un monde-pris-pour-donné (world-taken-for-granted), c'est à dire la croyance que demain sera comme aujourd'hui. Il faut souligner que dans le cadre de ce processus de délibération et de

27 On retrouve alors la perspective de G.H.Mead: l'apparition du nouveau ou du différent conduit à une reconstruction du monde social et à l'apparition d'un nouveau "soi" qui prend en compte cette nouveauté [voir Emirbayer 1997 p.309]

28 En ce sens l'entrepreunariat renvoie à une définition large correspondant à la mise en œuvre d'un processus d'enquête (au sens de Dewey) motivé par une insatisfaction, un doute, un déséquilibre perçu, ce qu'évoquait Mises quand il soulignait que tout le monde peut être entrepreneur [Loasby 2001 p.16].

36 Economie et Institutions $-\mathrm{n}^{\circ} 5-2^{\mathrm{e}}$ semestre 2004 
mise en œuvre d'un projet, la perspective développée par Schütz se rapproche de la théorie de l'action de Dewey. Celui-ci mettait en effet en avant le fait que les processus de décision et de délibération reposent sur un déséquilibre, une discontinuité, une insatisfaction...entre les cadres d'interprétation et d'action de l'individu (ses "habitudes") et la réalité qu'il expérimente, cela induit alors un processus de quête ou d'enquête [Dewey 1993 p.170]. A.Schütz citait ainsi fréquemment la phrase de Dewey issue de son ouvrage "Human nature and conduct" [1922]: "Deliberation is a dramatic rehearsal (in imagination) of various competing possible lines of action. [...]Deliberation is an experiment in finding out what the various lines of possible action are really like...But the trial is in imagination, not in overt fact"29. Pour Dewey [1916 p.319]: "[...] ideas as ideas are always working hypotheses concerning the attaining of particular empirical results, and are tentative programs (or sketches of method) for attaining them". De tels propos font écho à ce que disait L.Lachmann:"The business man who forms an expectation is doing precisely what a scientist does when he formulates a working hypotheses. Both business expectation ansd scientific hypotheses serve the same purpose: both reflect an attempt at cognition and orientation in an imperfecly known world, both embody imperfect knowledge to be tested and improved by later experience" [1997 p.90]. La proximité avec la perspective de Dewey est également très forte si on reprend l'analyse de Fu-Lai Yu [2003]. Ce dernier souligne en effet que dans le cadre du processus d'imagination (ou de délibération) chaque fin apparait comme un moyen pour une autre fin et que chaque plan est projeté au sein d'un système d'ordre ou de généralité supérieur. Dans une large mesure cela correspond au concept deweyen de "ends-in-view" et à sa considération des processus de choix comme une constante interaction entre des moyens et des fins qui ne sont jamais "donnés" mais imaginés, interprétés, inventés... et qui dépendant du contexte d'action et d'interprétation. Un autre point proche du pragmatisme est la considération que les individus ne s'adaptent pas passivement à l'environnement mais adaptent également leur environnement à eux, ce qu'avait évoqué Hayek dans "The sensory order"30. Les individus sont ainsi créateurs et utilisateurs de connaissances et sources du changement ${ }^{31}$.

\section{-La créativité de l'agir}

29 Voir Mousavi et Garrison [2003 p.145] et Musolf [2001]. Dans la perspective autrichienne voir Koppl [2001 p.184]

30 Voir Fu-Lai Yu [2003].

31 I.Kirzner soulignait ainsi la différence majeure de l'analyse autrichienne de la décision et des processus de choix par rapport aux "modèles de portefeuille" manipulés par l'analyse néo-classique, il mettait l'accent sur les qualités d'imagination, d'ouverture, de surprise...intrinsèques à ces processus [1997 p.64]

Economie et Institutions $-n^{\circ} 5-2^{e}$ semestre 2004 
La perspective pragmatique qui sous tends l'approche institutionnaliste partage de nombreux éléments avec ce qui précède et insiste également sur la "créativité de l'agir" pour reprendre les termes de H.Joas. Ainsi, comme l'ont souligné Beckert [2002] et Joas et Beckert [2002], les conceptions téléologiques de l'action impliquées par l'approche rationnelle ont du mal à traiter les questions de la création et de l'innovation. En effet, dans une large mesure, elles mettent en relation des moyens spécifiés avec un objectif spécifié. En ce sens le processus créatif est réduit à un calcul d'optimisation et se fonde sur une combinaison nouvelle d'élément préexistants et surtout connus, ce que Kirzner [1997] mettait également en avant. Il y a ainsi un paradoxe intrinsèque dans cette appréhension de la création de connaissances et de l'innovation: "(...)optimal strategies for innovative activities could only be devised if we would know at the outset what the innovation is. But if we know the innovation there is no need for innovation anymore" [Joas et Beckert 2002 p.4].

Dans une large mesure cette conception de l'innovation conduit à matérialiser les processus de création et d'innovation comme le développement d'un plan structuré d'action qui guide les actions et activités de l'innovateur. L'état final connu (la nouvelle "combinaison") oriente l'activité intentionnelle de conception. Ce type d'approche, qui ne pourrait être applicable, et encore, qu'à de pures activités d'ingénieur, ne rend pas compte du caractère fluent, mouvant, émergent...de tout processus de création de connaissance et d'innovation. Ici encore le contexte et son interprétation jouent un rôle important.

Cette conception de l'innovation et de la création trouve son origine dans une appréhension limitative de l'action que nous avons déjà évoquée, la conception interactionnelle. Une telle conception répond à une logique d'incitations, une logique en terme de stimulus et de réponse (le schéma S-R du behaviorisme mécaniste). Dans son article classique de 1896 "The reflex arc concept in psychology", J.Dewey a montré qu'un acteur ne réagit pas passivement à son environnement ou aux incitations qu'il reçoit. Au contraire l'acteur s'approprie son environnement de façon intentionnelle, et cela sur la base d'images ou de croyances. Une image est un produit de l'imagination mais n'est pas purement un reflet de la réalité [Khalil 2002 p.3]. L'imagination, et la création d'une image, mettent en œuvre une interprétation créative de l'environnement et la projection sur celui-ci des "fins-visées" (ends-in-view) par l'acteur. Pour reprendre ce que nous avions dit initialement: dans le cadre d'une conception pragmatique ou transactionnelle il n'y a pas de séparation entre le connu et le connaissant, entre le sujet et l'objet.

38 Economie et Institutions $-n^{\circ} 5-2^{e}$ semestre 2004 
La réalité est toujours prise en compte par l'acteur comme une représentation de la réalité, représentation médiatisée par les déterminations mentales, sociales, culturelles de l'acteur, ce qu'avait souligné également Hayek. Dans le même ordre d'idée G.L.S.Shakle a beaucoup insisté sur le fait que toute décision implique un élément de créativité puisqu'il ne peut y avoir de pure répétition du fait de la complexité du monde. Le processus créatif implique la création d'images des événements futurs pronostiqués permettant de guider l'action. C'est cet aspect qui est capturé par le concept deweyen de "fin-visée". L'action est conçue comme une quête ou une enquête c'est à dire une transaction entre le sujet connaissant (l'acteur avec ses croyances) et le connu (l'environnement, les "incitations"). Contrairement à la conception rationnelle dans laquelle, pour simplifier, l'environnement, les moyens, les fins, sont clairement spécifiés, la conception transactionnelle conçoit la réalité comme vague, indéterminée et dans une relation permanente de codétermination avec l'acteur. La spécification des moyens et des fins est donc toujours contingente et mouvante [Khalil 2002].

Pour Beckert [2002] l'ambiguïté intrinsèque des situations d'action ne peut être surmontée que dans le cadre d'un processus d'interprétation, et de communication, au terme duquel les agents se mettent d'accord sur la nature de la situation et adoptent une "définition commune de la situation"32: "The process of innovation depends on the social interpretation of the situation by the actors involved. Interpretations of the situation generate strategies of action, which are understood as adequate and thus guide action. What is valid especially in networks of organizations, but also in industrial regions like silicon valley, is that innovative processes progress without a clearly defined direction and this is crystallized only in the process of innovation itself" [Beckert 2002 p.275]. Il est d'ailleurs remarquable que de nombreux auteurs ayant tenté d'aborder sous l'angle de la créativité les processus d'innovation et de création de connaissance se soient référés à un cadre d'analyse issu de la phénoménologie ou du pragmatisme dont nous avons souligné la proximité en terme de problématique. C'est le cas par exemple de Shackle, Piore, Giddens, Luhmann, Schön...Malgré cela, comme le met en évidence Beckert, le pragmatisme qui constitue une tradition interprétative a été relativement peu utilisé, à quelques exceptions près, alors même que la pertinence de cette démarche pour appréhender la créativité de l'agir a été démontré par Joas [1999 (1992)], celui-ci se référant aux travaux de J.Dewey et G.H.Mead. De même, chez C.S.Peirce, le fondateur du pragmatisme, il y a

32 B.J.Loasby, dans une perspective autrichienne a également souligné le caractère potentiellement générateur de nouveauté de l'ambiguïté [2001 p.9]

Economie et Institutions $-n^{\circ} 5-2^{e}$ semestre 2004 
clairement la volonté de mettre en avant le caractère inter-subjectif de l'usage de signes et le rôle de la créativité dans la formulation d'hypothèses scientifiques ${ }^{33}$, ce qui renvoie également aux propos de L.Lachmann ${ }^{34}$. Ainsi, à côté des processus usuels de la démarche scientifique que sont l'induction et la déduction, C.S.Peirce avait défini l'abduction comme le production d'hypothèses nouvelles dans un acte créateur. La démarche de production de connaissance constitue donc un acte de création qui peut être apparenté au sens étymologique à une démarche "poétique" (ou poïetique), qui n'est pas réservée au domaine de la science. Le langage, l'usage de signes, la communication...jouent un rôle essentiel puisque nous pensons en terme de mots et de symboles ${ }^{35}$. Ce type de processus abductif est évidemment particulièrement prégnant dans les communautés informelles orientées vers la production et la diffusion de connaissances [Diani 2004, Cohendet et Diani 2003].

La conception pragmatique de l'action permet aussi de mettre en parallèle deux éléments largement présents dans les théories économiques des processus d'innovation et de production de connaissances, par exemple les approches évolutionnistes:

-d'un côté la perception du monde et l'action sont modelés par des routines, des habitudes d'action, qui permettent de stabiliser l'action et agissent comme une grammaire sociale permettant l'intercompréhension et la coordination [Renault 1997,1999], ce qu'on retrouve également chez les "conventionnalistes".

-d'un autre côté cette "grammaire sociale" est soumise à des processus interprétatifs permanents et n'est pas seulement prescriptrice mais également génératrice. Cela correspond à ce que disait G.H.Mead: "The appearance of different interests in the form of reflection [leads to] the reconstruction of the social world, and the consequent appearance of the new self that answers to the new

33 voir aussi Joas 1999 p.144

34 On comprend alors la pertinence de la référence au pragmatisme pour aborder ce qu'il est convenu d'appeler la "cognition encastrée" (embedded cognition), qui a donné lieu à un colloque récent (BRC, Great Barrington juillet 2003) mettant en parallèle les approches de Dewey et Hayek, colloque auquel ont participé deux économistes aussi éminents que D.North ou V.Smith.

35 R.Stacey [2000 p.37] se référant à Mead et à Schütz soulignait les dimensions communicationnelles de la connaissance: "Knowledge is the act of conversing and new knoledge is created when ways of talking, and therefore patterns of relationship, change" et "Organizational change, learning and knowledge creation are the same as change in communicative interaction, whether people are conscious of it or not". Les travaux de M.Zacklad [2004] se placent dans une perspective similaire.

40 Economie et Institutions $-n^{\circ} 5-2^{e}$ semestre 2004 
object" [Mead 1964 p.149], ce qui est proche de la conception de l'esprit de Hayek et de celle de Schütz. La créativité permet ainsi de remettre en cause les habitudes et les routines, en particulier dans le cas où elles ne permettent pas d'interpréter correctement une situation ou sont incapable de faire face au nouveau, à l'imprévu, à la nécessité d'adaptation. La notion de "créativité situationnelle" mise en avant par H.Joas met en évidence le fait que les acteurs ne sont pas limités par un ensemble préexistant d'alternatives d'action. La résolution de problèmes émerge dans le cadre de l'interaction entre la situation et les processus cognitifs, cette résolution demande toujours une interprétation de la situation par les agents. Il y a donc une conception procédurale et situationnelle de la résolution de problèmes et de la création de connaissances. Ainsi, comme l'affirme H.Joas [1999 p.143): "Chaque situation présente, aux yeux des pragmatistes, un horizon de possibilités pratiques, et c'est cet horizon qui, dans les moments de crise, doit être redéployé. Des hypothèses sont avancées; nous jetons de nouvelles passerelles entre nos propres impulsions et les données extérieures. Toutes ces passerelles ne sont pas solides. Mais si nous parvenons à en établir une, alors nous avons concrètement enrichi notre capacité d'action. Celle-ci modifie jusqu'aux fins que s'assigne le sujet agissant. (...) Ainsi ancrée dans l'action, la créativité apparaît (...) comme une ouverture à de nouvelles façon d'agir" (nous soulignons). La pertinence de cette approche pour appréhender les processus de conception de nouveaux produits a ainsi été soulignée par C.Sabel ou D.Schön [voir Beckert 2002 p.279].

\section{-Entrepreunariat et images}

Nous avons souligné que dans le cadre d'une conception pragmatique de l'action, le schéma rationaliste moyens-fins est remplacé par une conception transactionnelle dans laquelle les fins de l'action, les moyens et les résultats se codéterminent au sein de processus interprétatifs permanents, ce qui est capturé par le concept de "situation" [Beckert 2002 p.276]. De même l'approche de H.Joas [1999] a réinterprété la position de J.Dewey qui repose sur la conviction que l'on peut intentionnellement modeler les mondes de la vie sociale dans le cadre de processus comunicationnels: "The undetermined future, this is the basic idea, is shaped on the basis of ideas, plans, theories and normative thoughts and is thus always grasped in an unfinished process of becoming" [Beckert 2002 p.276]. Le concept d'image s'inscrit dans une telle perspective. En effet, la notion de "situation" que nous avons évoquée plus haut ne correspond pas à un élément externe purement objectif. Une situation n'est abordée que via une "définition de la situation", concept développé par W.I.Thomas, qui ne peut être que subjective 
puisque dépendant des perceptions et interprétations de l'acteur. Une telle intuition se retrouve chez K. Boulding [1956] pour lequel la connaissance trouve son origine et s'incarne dans des "images" qui sont propres à chaque individu et orientées vers le futur. De telles "images" dépendent du présent et de son interprétation par l'acteur, de la "situation" ainsi que des valeurs et des objectifs de l'individu [Fazio 2003 p.24]. Le processus d'élaboration de ces images est fondamentale pour la compréhension des processus cognitifs mais également pour celle de l'ensemble des activités humaines y compris l'activité productive. D'autre part, bien que cela ne soit pas développé par Boulding, il faut souligner que les images impliquent nécessairement une dimension réflexive et intersubjective comme cela a été souligné par Mead et plus récemment par Joas [1999] et Beckert [2002]. En ce sens, le concept d'"entrepreneur" peut être élargi dans une perspective pragmatique. En effet, selon J.Dewey l'action s'enracine dans une rupture de l'équilibre, une insatisfaction, une situation problématique...que l'acteur cherche à dépasser. Trouver une réponse, une solution, à une situation problématique implique un acte d'imagination qui donnera naissance à un plan d'action, éventuellement révisé, si l'action échoue ou se révèle insatisfaisante. En ce sens, tout acteur cherchant à résoudre un problème ne peut se satisfaire d'un comportement purement routinier, il doit "entreprendre" un processus de cognition et d'action, chercher de nouvelles opportunités, élargir son horizon de possibilités...Il apparaît en ce sens comme un "entrepreneur" au sens autrichien. Pour E.Khalil "Entrepreneurship is not only about innovation or the management of an enterprise, although innovation or management are instances of entrepreneurship. Entrepreneurship, as I define it here, is nothing other than action per se-where action can be challenging, which is then called entrepreneurship in the strict sense, or less challenging, which is called entrepreneurship in general. That is, action by definition involves challenge and creativity [Joas 1996] although in different degrees. (...) Entrepreneurship is simply action where the means and ends form a bundle that cannot be analytically separated" [2003 p.170-nous soulignons] ${ }^{36}$.

\section{-L'apprentissage organisationnel}

L'approche de J.R.Commons reflète cette conception pragmatique de l'action et de la création. Il soulignait ainsi qu'au sein d'organisations les agents agissent selon des règles d'action (working rules). Ces règles permettent de faciliter les anticipations et

36 Cela correspond également à ce que développe U.Witt quand il parle d'imagination entrepreneuriale et de l'importance de la communication [Witt 1999]

42 Economie et Institutions $-\mathrm{n}^{\circ} 5-2^{\mathrm{e}}$ semestre 2004 
les actions communes en déterminant quels choix sont possibles dans une situation, ce que Commons appelle un "champ d'opportunité"; la coordination des actions est donc largement simplifiée. Il faut voir en retour que les individus peuvent contribuer à la modification des règles d'action: "(...)the field of opportunity allows the individual a wider array of practices than those traditionnally followed-some untested, some not even imagined. Some, not all, individuals will continually seek to expand their field of opportunity, searching for new activities which are permissible under existing definition of rights and liberties and which allow them a differential advantage over others. In Commons's terminology their active minds are searching for a "limiting factor" upon which they can act "[Biddle 1990 p.23]. Ce rôle ressemble ainsi à celui joué par l'entrepreneur de l'approche autrichienne.

Dans ce cadre, l'apprentissage organisationnel peut être définit de façon simple comme un processus qui change les règles et pratiques d'une organisation [Lorenz 2000 p.201]. L'approche de Commons peut alors être créditée pour surmonter la dichotomie usuelle entre le changement comme processus délibéré et le changement comme processus spontané. En effet, une telle dichotomie, un tel dualisme, est étranger au mode de raisonnement pragmatique. Comme le souligne Commons, les institutions contraignent le comportement mais contribuent également à le libérer. Cette libération, ce caractère permissif des institutions a été souvent référé à juste titre à la sécurité des transactions et des anticipations qu'elles génèrent. Un autre aspect peut selon nous être ajouté: si on envisage les institutions comme une "grammaire sociale", on doit considérer que celle-ci est aussi potentiellement génératrice et pas seulement prescriptrice. En effet comme l'affirmait G.H.Mead [1963 p.169]: "il existe un langage commun, mais chacun peut en faire un usage différent dans chaque nouveau contact avec autrui. Il y a un élément de nouveauté dans une telle reconstruction grâce à la réaction du groupe dont ils font partie". R.M.Dawson [1994] a ainsi souligné la proximité entre la notion de valeur "raisonnable" de Commons et l'approche de G.L.S.Shackle ${ }^{37}$, la capacité de prise de rôle et l'anticipation par l'imagination du futur étant des points communs. Un des rôle des "courts de justice" ou des "officiels" de la collectivité est donc d'institutionnaliser les innovations, les nouvelles interprétations ou définitions d'une situation, qui émergent des interactions si celles ci se révèlent "fonctionnelles" et/ou sont soumises à un processus de généralisation et de diffusion qui, à

37 Les liens de G.L.S.Shackle avec le courant autrichien sont ambigus mais revendiqués par certains auteurs. Le "raisonnable" chez Commons renvoie à un processus de discussion, de conversation...

Economie et Institutions $-\mathrm{n}^{\circ} 5-2^{\mathrm{e}}$ semestre 2004 
partir de conventions aboutit à de nouvelles coutumes ou routines. C'est ce que Commons appelle une méthode de Common law ("Custom becomes common law by the common law method" [1934 p.707]). Ces aspects sont capturés par deux citations de Commons reprises par E.Lorenz:

-"For custom is the mere repetition, duplication, and variability of practices and transactions. No repetition is exactly the same as its contemporary. Hence, there is always a variability of customs in successive times and at the same time. These variations in the course of history introduce new customs as variables or as alternatives, of proceedings or contemporary customs" (nous soulignons)

-"The practices of any individual, or firm or association may be variable and indifferent to others because not sufficiently imitated to induce general imitation, as when one person practices economy and the other extravagance. But a usage becomes sufficiently imitated, so that, like language, or bank checks, its use is practically compulsory on all who participates in transactions (nous soulignons)"[1934 p.709].

Chez Commons, l'évolution des règles d'action possède donc un aspect spontané, mais elle revêt aussi un aspect dirigé. En effet, les individus agissant au sein d'organisations sont soumis au pouvoir, à la souveraineté. Comme le souligne Commons, face à de nouvelles règles ou activités, les "officiels" de la collectivité altèrent les règles d'action pour développer et généraliser la réponse ou l'innovation qui sert le mieux l'objectif collectif commun [Commons 1950 p.185 et ss.]. Nous sommes alors dans le domaine de la "sélection artificielle". Ainsi pour Commons: "(the) artificial selection which we name institutionalism (...) is the evolution of control of individuals by collective action. Its product in individual has been named the institutionalized mind" [1950 p.192]. Ces propos ne peuvent être compris sans référence aux processus cognitifs qui les sous tendent.

Dans cette perspective il nous semble que l'on peut rapprocher cette conception de celle développée par U.Witt que nous avons déjà évoquée. En effet si on prend comme définition du leadership (dans l'approche de Commons on pourrait parler de souveraineté) celle que développe U.Witt [1998 p.167], c'est à dire le fait de réussir à faire prédominer ses interprétations, représentations et définitions sur celles des autres, ce qui implique de faire en sorte qu'elles soient imitées et diffusées plus largement. Dans ce cadre: "Leadership of this kind can be attributed more precisely to traits that help to win through informal communication, such as eloquence,

44 Economie et Institutions $-\mathrm{n}^{\circ} 5-2^{\mathrm{e}}$ semestre 2004 
persuasiveness, patience and persistance, the capacity for generating sympathy and confiance" 38 . Chez Commons, le processus transactionnel est envisagé comme un processus de communication et exhibe des caractéristiques rhétoriques de persuasion, d'influence...qui aboutit à une définition commune de la réalité, ce qui n'empêche pas la domination d'une conception sur une autre. Toute organisation est le siège de processus de socialisation qui prennent place par l'intermédiaire de la communication, ce que souligne également U.Witt [1998 p.167, 1999 p.102]. Les communautés intensives en connaissance sont évidemment particulièrement perméables à ce genre de phénomènes. Dans un tel cadre, les approches fondées sur des hypothèses comportementales telles que l'opportunisme et la poursuite de l'intérêt individuel sont insuffisantes. D'une certaine façon, elles ne traitent réellement que des cas où les acteurs ne partagent pas de cadres communs d'interprétation et négligent donc les processus de socialisation à l'œuvre dans tout système social. Comme le dit U.Witt: "(...) if opportunism were indeed the only mode of behavior, and tight governance and monitoring effort the only response, there could never be something like a creative organization" [1998 p.173]. Si les membres d'une organisation partagent une vision entrepreneuriale et se l'approprient ils seront d'autant moins enclins à développer des conduites opportunistes. Le partage d'une telle conception ou vision modèle en effet les mécanismes de "prise de rôle" 39 , essentiels dans les processus d'interaction et de communication. Le partage de telles "images "est particulièrement apparent dans le cadre des communautés, ce que tendent à montrer des approches récentes.

\section{Conclusion}

Les approches institutionnalistes et autrichiennes n'ont pas, a priori, proposé de théorie propre de la firme ou de l'organisation. Cependant, de nombreux éléments analytiques portent en germe la possibilité de développements permettant d'enrichir la compréhension que les économistes ont des firmes et des organisations. Ces dernières années ces approches ont fait l'objet

38 T.Fu-Lai Yu [2003 p.253], se plaçant dans la perspective de Schütz, écrit dans cet ordre d'idée: "By establishing a firm, the entrepreneur is in fact building a coherent world of knowledge and a cultural community"

39 Tout système social, en particulier une firme, implique la réciprocité, ce qui a été évoqué par A.Schütz dans son appréhension de la coordination. La réciprocité [Bange 1992 p.211] désigne le fait qu'une action sociale s'engrène toujours sur l'action sociale d'un autre acteur. Dans ce cadre, chacun à la capacité de "prendre le rôle" de l'autre. Cette réciprocité implique en particulier la réciprocité des "images": les acteurs partagent une représentation de leurs situation d'interaction.

Economie et Institutions $-\mathrm{n}^{\circ} 5-2^{\mathrm{e}}$ semestre 2004 
d'un regain d'intérêt qui, nous semble t-il, est redevable avant tout à la prise en compte des phénomènes liés à la connaissance, à l'apprentissage, à la coordination, à la formation de routines, de modèles mentaux partagés...qui sont au cœur de nombreuses recherches actuelles. Ces approches nous semblent ainsi mériter d'être développées afin de clarifier et préciser leurs intuitions analytiques; l'enjeu principal étant celui de leur opérationalisation. D'autre part, des phénomènes important tels que le pouvoir, la souveraineté, la négociation autour des règles et des transactions mériteraient plus d'attention puisqu'ils sous tendent également, conjointement avec les aspects cognitifs, les phénomènes institutionnels et organisationnels. Pour notre part il nous semble également que les aspects communicationnels des interactions et de la formation de cadres communs de pensée et d'action, qui apparaissent en filigrane de ces approches, devraient également retenir l'attention.

\section{REFERENCES}

Albert A. \& Ramstad Y. (1997) "The social psychological underpinnings of Commons's Institutional Economics : the significance of Dewey's Human Nature and Conduct "Journal of Economic Issues, Vol.31 n4-décembre, (pp.881-916).

Albert A. \& Ramstad Y. (1999) "The social psychological underpinnings of Commons's Institutional Economics II : the concordance of G.H.Mead's "Social self" and J.R.Commons's "Will " " Journal of Economic Issues, Vol.33 n4-Mars, (pp.1-46).

Atkinson G. \& Reed M.(1992) "The individual in a going concern" Journal of Economic Issues, Vol.26 n 2 june, (pp.469-476).

Bange P. (1992) "Analyse conversationnelle et théorie de l'action", Hatier, Paris

Bazzoli L \& Dutraive V (1998a) "Les dimensions cognitives et sociales $\mathrm{du}$ comportement humain: l'approche institutionnaliste de J.R.Commons" Cahiers du Gratice, ${ }^{\circ} 14$

Bazzoli L \& Dutraive V (1998b) "Une conception institutionnaliste de l'organisation comme institution. Eléments sur l'apport de J.R.Commons" Communication aux journées d'étude "Institutions et organisations", Amiens, 13 mai

Beckert J. (2002) "Beyond the market-The social foundations of economic efficiency" Princeton University Press, Princeton \& Oxford Beckert J. (2003) "Economic sociology and embededness: how shall we conceptualize economic action" Journal of Economic Issues, Vol $37 \mathrm{n}^{\circ} 3$ september, (pp.769-787)

Bensaïd M. \& Richebé N. (2001) "Règles d'organisation et relation salariale" Revue d'Economie Industrielle n97 4ème trimestre (pp.6984)

46 Economie et Institutions $-n^{\circ} 5-2^{e}$ semestre 2004 
Berger P. et Luckmann T. (1966) "The social construction of reality" Anchor Books, New York

Biddle Jeff E.(1990) "The role of negotational psychology in J.R.Commons's proposed reconstruction of political economy" Review of Political Economy, ${ }^{\circ} 2$ march, (pp.1-25)

Boettke P.J. (1989) "Evolution and economics: Austrians as institutionalists" in Samuels W.J. (ed.) Research in Economic Thought and Methodology, Vol.6, Elsevier, (pp.73-90)

Boettke P.J. (2002) "Information and knowledge: Austrian economics in search of its uniqueness" The Review of Austrian Economics, 15:4, (pp.263-274)

Boltanski L.,Thévenot L.(1991) "De la justification-Les économies de la grandeur" Gallimard, Paris

Chavance B. (2001) "Organisations, institutions, systèmes: types et niveaux de règles" Revue d'Economie Industrielle $n^{\circ} 97$ 4ème trimestre (pp.85-102)

Cohendet Patrick et Diani M. (2003) "L'organisation comme une communauté de communautés: croyances collectives et culture d'entreprise" Revue d'économie politique, $\mathrm{n}^{\circ} 5$, (pp. 697)

Commons J.R.(1974) "Legal foundations of capitalism" (1924)

A.M.Kelley, Clifton

Commons J.R (1931) "Institutional economics" American Economic Review Vol.21 n³, december, (pp.648-657)

Commons J.R.(1934) "Institutional economics» McMillan, New York

Commons J.R.(1936) "Institutional economics" American Economic Review Vol.26 n ${ }^{\circ}$, march, (pp.237-249)

Commons J.R.(1950) "The economics of collective action" McMillan, New York

Coriat B. \& Weinstein O. (1995) "Les nouvelles théories de l'entreprise" Le Livre de Poche (Références), Paris

Cosgel M., Langlois R.N.(1998) "The organization of consumption " in "The active consumer "Ed. par M.Bianchi, Routledge, London, New York (pp.107-121).

Dawson R.M. (1994) "The Shacklean nature of Commons's reasonable value" Journal of Post Keynesian Economics, Vol.17 nº 1 , (pp.33-44)

Denzau A.T. \& North D.(1994) "Shared mental models: ideologies and institutions" Kyklos, Vol.47 Fasc. 1, (pp.3-31)

Dewey J.(1922) «Human nature and conduct» Henry Holt \& Co., New York

Dewey J.(1958) "Experience and nature» (1929) Dover Publ., New York

Dewey J.(1975) "Démocratie et éducation»(1916) A.Colin(Coll.U), Paris

Dewey J.(1993) «Logique, la théorie de l'enquête» (1938) PUF, Paris 
Dewey J. \& Bentley A.F. (1973) "Knowing and the known" (1949) in Rollo Handy \& E.C.Harwood (Eds.) "Useful procedures of inquiry" Behavioral Research Council, Great Barrington (Mass). Disponible en version électronique: www.brc-aier.org

Diani M (2002) "Economie basée sur la connaissance, organisations et nouvelles formes de coordination" colloque "La métamorphose des organisations" Nancy-Vittel 23-25 octobre

Diani M (2004) "Les communautés intensives en connaissance comme nouvelle forme de coordination efficace dans les organisations" in M.Baslé et M.Renault Eds. "L'économie fondée sur la connaissance", Economica, Paris, (pp.122-141)

Dulbecco P. (1998) "La dimension historique du temps dans une théorie néo-autrichienne de la firme et de l'organisation" Economie Appliquée tome LI n ${ }^{\circ} 1$ (pp.53-76)

Dulbecco P. et Garrouste P. (2000) "Structure de la production et structure de la connaissance" Revue Economique, Vol.51 n ${ }^{\circ} 1$-janvier, (pp.75-103),

Dulbecco P. et Dutraive V. (2001) "The meaning of market: comparing Austrian and institutional economics" in "Evolution and path dependance in economic ideas" Ed. par S.Ioannidès et P.Garrouste, E.Elgar, Aldershot

Dutraive V. (1993) "La firme entre transaction et contrat: Williamson épigone ou dissident de la pensée institutionnaliste" Revue d'Economie Politique, Vol. $103 \mathrm{n}^{\circ} 1$ janvier-février , (pp.83-103)

Emirbayer E. (1997) "Manifesto for a relational sociology", American journal of Sociology, Vol.103 n², September, (pp.281-317)

Favereau O.(1989a) "Marchés internes, marchés externes" Revue Economique, Vol.40 $\mathrm{n}^{\circ} 2$, mars (pp.275-328).

Favereau O. (1989b) "Organisation et marché" Revue Française d'Economie Vol.IV n 1 (pp.65-96)

Forest J. et Méhier C. (2001) "J.R.Commons and H.A.Simon on the concept of rationality" Journal of Economic Issues, Vol.35 $\mathrm{n}^{\circ} 3$, (pp.591-605)

Foss N.J. (1994) "The theory of the firm: the austrians as precursors and critics of contemporary theory" Review of Austrian Economics, Vol. $7 \mathrm{n}^{\circ} 1$, (pp.31-65)

Foss N.J. (1996a) "Spontaneous social order: Economics and Schützian sociology" American Journal of Economics and Sociology, Vol.55 n 1 , (pp.73-86)

Foss N.J. (1996b) "T.B.Veblen: precursor of the competence-based approach to the firm" DRUID Working Paper n ${ }^{\circ} 96-15$

Foss N.J. (1997) "Austrian economics and the theory of the firm" DRUID Working Paper $n^{\circ}$ 97-3

Foss N.J. (1998a) "Firms and the coordination of knowledge: some austrian insights" DRUID Working Paper n 98-19

48 Economie et Institutions $-n^{\circ} 5-2^{e}$ semestre 2004 
Foss N.J. (1998b) "Market process economics and the theory of the firm" Working paper

Foss N.J. (2003) "Bounded rationality in the economics of organizations: "much cited and little used"" Journal of Economic Psychology, 24, (pp.245-264]

Foss K. \& Foss N.J. (2000) "Theoretical isolation in contract theory: suppressing margins and entrepreneurship" Journal of Economic Methodology, Vol.7 n³, (pp.313-339)

Fu-Lai Yu T.(1999) "Toward a praxeological theory of the firm" Review of Austrian Economics, vol. $12 \mathrm{n}^{\circ} 1$, (pp.25-41)

Fu-Lai Yu T.(2001) "Entrepreneurial alertness and discovery" Review of Austrian Economics" vol. $14 \mathrm{n}^{\circ} 1$ (pp.47-63)

Fu-Lai Yu T.(2003a) "Innovation and coordination: a Schützian perspective" Economic innovation and New Technologies, Vol.12 $\mathrm{n}^{\circ} 3$, Octobre (pp.397-412)

Fu-Lai Yu T.(2003b) "National capabilities and economic development: a subjectivist view" Forum for Development Studies, $\mathrm{n}^{\circ} \mathrm{é}$ (pp.248-267)

Garrouste P. (1995) "La firme entre institution et organisation: une solution au problème hayékien de la connaissance dispersée" Colloque "La connaissance dans la dynamique des organisations productives", Aix-en-Provence 14-15 septembre

Garrouste P. (1999) "La firme "hayékienne" entre institution et organisation" Revue d'Economie Politique, vol.109 $\mathrm{n}^{\circ} 6$, novembredécembre , (pp.885-902)

Garrouste P. (2002) "Knowledge: a challenge for the austrian theory of the firm" ATOM Working Paper-repris dans "Entrepreneurship and the firm. Austrian perspectives on economic organizations" ed. par N.J.Foss et P.G.Klein, E.Elgar, Aldershot

Habermas J.(1987a) "Théorie de l'agir communicationnel» t.II Fayard, Paris

Habermas J.(1987b) «Explicitation du concept d'activité communicationnelle"(1982) in "Logique des sciences sociales et autres essais" PUF, Paris (pp.413-446).

Hamilton D.(1953) "Newtonian classicism and Darwinian institutionalism-A study of change in economic theory" The University of Mexico Press, Albuquerque

Hayek F.A. (1988) "The fatal conceit: the errors of socialism", Routledge, London

Hörwitz S. (2001) "From Smith to Menger to Hayek-Liberalism in the spontaneous order tradition" The independant review Vol.VI $n^{\circ} 1$ (pp.81-97)

Ioannidès S. (1999) "Marché, firme et direction d'entreprise: une perspective hayékienne" Revue d'Economie Politique vol.109 n6 novembre-décembre (pp.871-883) 
H.Joas (1985) "G.H.Mead: a contemporary reexamination of his thought" Polity Press, Cambridge

Joas H. (1999) "La créativité de l'agir" (1992) Editions du Cerf, Paris

Joas H. \& Beckert J. (2002) "A theory of action: pragmatism and the theory of action" Transactional Viewpoints, Vol. $1 \mathrm{n}^{\circ} 4$ automne, www.brc.aier.org

Kaisla J. (2002) "Choice behaviour: looking for remedy to some central logical problems in rational action" Kyklos, Vol.56 Fasc.2, (pp. 245-262)

Kaufman B.E. (1999) "Emotional arousal as a source of bounded rationality" Journal of Economic Behaviour and Organization, Vol 38, (pp.135-144)

Kaufman B.E. (2002) "The organization of economic activity: insights from the institutional theory of J.R.Commons" Journal of Economic Behaviour and Organization, Vol 15, (pp.1-26)

Khalil E.L. (2003) "A transactional view of entrepreneurship: a deweyan approach" Journal of Economic Methodology Vol.10 $\mathrm{n}^{\circ} 2$ Juin (pp.161-179)

Kilpinen E. (2003) "Does pragmatism imply institutionalism" Journal of Economic Issues, Vol.37 n², (pp.291-304)

Kirzner I. (1973) "Entrepreneurship and competition", University of Chicago Press, Chicago

Kirzner I. (1979) "Perception, opportunity and profit: studies in the theory of entrepreneurship", University of Chicago Press, Chicago

Kirzner I. (1997) "Entrepreneurial discovery and the competitive market process: an Austrian approach", Journal of Economic Litterature, Vol.35 n¹, (pp.60-85)

Klein P.G. (1996) "Economic calculation and the limits of organizations" Review of Austrian Economics, Vol. 9 n² , (pp.3-28)

Koppl R. (2001) "A.Schütz et G.Schakle: two views of choice", Review of Austrian Economics, 14: 2/3, (pp.181-191)

Koumakhov R. (2003) "Le paradigme H.Simon: un soutien pour l'analyse conventionnaliste de l'entreprise" Colloque "Economie de la firme: quelles nouveautés" Université de Savoie-IREGE, 17 et 18 avril Kurrild-Klitgaard P. (2003) "The Viennese connection: A.Schütz and the Austrian school" The Quarterly Journal of Austrian Economics, Vol.6 n 2 , (pp.35-67)

Lachmann L. (1970) "The legacy of M.Weber", Heineman, London

Lachmann L. (1977) "Pr. Shackle ont the economic significance of time" (1959) repris dans: Grinder W. ed. "Capital, expectations and the market process", Andrews et Mc Meel, Kansas City

Leathers C.G. (1989) "New and old institutionalists on legal rules: Hayek and Commons" Review of political Economy, Vol.1 $\mathrm{n}^{\circ} 3$, (pp.361-380)

50 Economie et Institutions $-\mathrm{n}^{\circ} 5-2^{\mathrm{e}}$ semestre 2004 
Lewin P. \& Phelan S.E. (2000) "An austrian theory of the firm" Review of Austrian Economics Vol.13 $\mathrm{n}^{\circ} 1$ (pp.59-79) Working Paper (www.utdallas.edu/ plewin/)

Loasby B.J. (2000) "The division and organisation of knowledge" European Journal of Economic and Social Systems, Vol. $14 \mathrm{n}^{\circ} 2$, (pp.143-155)

Loasby B.J. (2001) "Cognition, imagination and institutions in demand creation" Journal of Evolutionary Economics, 11, (pp.7-21)

Loasby B.J. (2004) "Hayek's theory of mind" in Advances in Austrian Economics, Vol.7, Elsevier, Amsterdam, (pp.101-134)

Longuet S. (2001) "La firme dans une perspective autrichienne" Revue d'Economie Industrielle, $\mathrm{n}^{\circ} 97$ 4ème trimestre, (pp.35-52)

Lorenz E. (2000) "Organizational routines in the light of "old" evolutionary economics: bringing politics back into the study of organizational learning" European Journal of Economic and Social Systems , $14 \mathrm{n}^{\circ} 2$, (pp.191-207)

Lorenz E. (2002) "Organisational routines and organisational learning in their cognitive and political dimensions" EURAM 2002 "Organisational learning and knowledge management", Stockholm, 9-11 mai

Mead G.H.(1963) "L'esprit, le soi, la société" (1934) PUF, Paris

Mead G.H.(1964) "The social self" in "G.H.Mead: selected writings" ed. par A.J.Reck, Bobbs-Merril, Indianapolis (pp.142-149)

Mote J.E. (2001) "From Schütz to Goffman: the search for social order" Review of austrian Economics, Vol.14 n²-3, (pp.219-231)

Mousavi S., Garrison J. (2003) "Toward a transactional theory of decision making: creative rationality as functional coordination in context", Journal of Economic Methodology, 10 :2, June, pp.131-156 Musolf G.R. (2001) "J.Dewey's social psychology and neopragmatism: theoretical foundations of human agency and social reconstruction" The Social Science Journal, 38, (pp.277-295)

Oakley A. (2000) "A.Schütz and economics as a social science" Human Studies, 23, (pp.243-260)

O'Driscoll \& Rizzo M. (1985) "The economics of time and ignorance" Basil Blackwell, Oxford

Prendergast C. (1986) "A.Schütz and the austrian school of economics" American Journal of Sociology, Vol.92 n¹, (pp.1-26)

Ramstad Y.(1986) "A pragmatic quest for holistic knowledge:the scientific methodology of J.R.Commons" Journal of Economic Issues, Vol.20 $\mathrm{n}^{\circ} 4$ décember, repris dans "Institutional Economics" (W.J.Samuels Ed.) (pp.138-176)

Ramstad Y.(1994) "On the nature of economic evolution: J.R.Commons and the metaphor of artificial selection" in "Evolutionary and neo-schumpeterian approaches to economics" Ed. by L.Magnusson, Kluwer, Boston-Dordrecht-London (pp.65-121) 
Renault M.(1992) "L'économique institutionnaliste et la philosophie pragmatique: la nature humaine, les totalités et les valeurs" Economies et Sociétés série PE Oeconomia, n¹7-8, (pp.171-201)

Renault M. (1997) "Economie et communication: une approche institutionnaliste des conventions et des institutions " Economies et Sociétés, série R n¹0-décembre, (pp.48-95).

Renault M. (1999) "Economie et coordination des comportements: communication et interaction", Revue Européennes des Sciences Sociales, Tome 37, n¹14, (pp.265-292)

Salais R. \& Storper M. (1993) "Les mondes de production " Editions de l'EHESS, Paris.

Samuels W.J. (ed.) (1989) Research in Economic Thought and Methodology, Vol.6, Elsevier, Amsterdam

Shalin D.N. (1986) "Pragmatism and social interactionism", American Sociological Review, Vol.51 n 1 february, (pp.9-29)

Scheff T.J.(1993) "Toward a sociological psychological theory of mind ans consciousness" Social Research, Vol $60 \mathrm{n}^{\circ} 1$, Spring, (pp.171196)

Schütz A. (1964) "The social world and the theory of social action" in Schütz A. "Collected papers II" (pp.3-19), M.Nijhoff, La Haye

Schütz A. (1967) "The problem of social reality: Collected papers Vol.1", M.Nijhoff, La Haye

Schütz A. (1987) "Le chercheur et le quotidien" MéridiensKlincksieck, Paris

Schütz A. et Luckmann T.(1989) "The structures of the life world" Vol.2, Northwestern University Press, Evanston

Stacey R. (2000) "The emergence of knowledge in organizations", Emergence, Vol.2 $\mathrm{n}^{\circ} 4$ (pp.23-39)

Ughetto P. (2000) "L'entreprise dans l'économie des conventions" Revue Economique Vol.51 n 1 -janvier (pp.51-73)

Vanberg V.(1989) "Carl Menger's evolutionary and J.R.Commons' collective action approach to institutions: a comparison" Review of Political Economy n ${ }^{\circ} 1$ (3) November (pp.334-360).

Vanberg V.(1992) "Organizations as constitutional systems" Constitutional Political Economy Vol. $3 \mathrm{n}^{\circ} 2$ (pp.223-253)

Witt U. (1998) "Imagination and leadership. The neglected dimension of an evolutionary theory of the firm" Journal of Economic Behaviour and Organization" Vol.39 n 2 (pp.169-178)

Witt U. (1999) "Do entrepreneurs need firms ? A contribution to a missing chapter in Austrian economics" Review of Austrian Economics, 11, (pp.99-109)

Zacklad M. (2004) "Transactions communicationnelles symboliques et communauté d'action : une approche de la création de valeur dans les processus coopératifs" actes du Colloque de Cerisy, La Découverte, Paris

52 Economie et Institutions $-\mathrm{n}^{\circ} 5-2^{\mathrm{e}}$ semestre 2004 